\title{
An Introduction to Chiral Symmetry on the Lattice
}

\author{
S. Chandrasekharan ${ }^{1}$ and U.-J. Wiese ${ }^{2}$ \\ ${ }^{1}$ Department of Physics, Duke University, Durham, North Carolina, U.S.A. \\ ${ }^{2}$ Institute for Theoretical Physics, Bern University, Bern, Switzerland
}

February 1, 2008

\begin{abstract}
The $S U\left(N_{f}\right)_{L} \otimes S U\left(N_{f}\right)_{R}$ chiral symmetry of QCD is of central importance for the nonperturbative low-energy dynamics of light quarks and gluons. Lattice field theory provides a theoretical framework in which these dynamics can be studied from first principles. The implementation of chiral symmetry on the lattice is a nontrivial issue. In particular, local lattice fermion actions with the chiral symmetry of the continuum theory suffer from the fermion doubling problem. The Ginsparg-Wilson relation implies Lüscher's lattice variant of chiral symmetry which agrees with the usual one in the continuum limit. Local lattice fermion actions that obey the Ginsparg-Wilson relation have an exact chiral symmetry, the correct axial anomaly, they obey a lattice version of the Atiyah-Singer index theorem, and still they do not suffer from the notorious doubling problem. The Ginsparg-Wilson relation is satisfied exactly by Neuberger's overlap fermions which are a limit of Kaplan's domain wall fermions, as well as by Hasenfratz and Niedermayer's classically perfect lattice fermion actions. When chiral symmetry is nonlinearly realized in effective field theories on the lattice, the doubling problem again does not arise. This review provides an introduction to chiral symmetry on the lattice with an emphasis on the basic theoretical framework.
\end{abstract}




\section{Introduction}

Physical phenomena arise over a vast range of energy scales. Attempts to unify gravity with the other fundamental forces suggest that the Planck scale

$$
M_{P}=\frac{1}{\sqrt{G}} \approx 10^{19} \mathrm{GeV}
$$

which is constructed from Newton's constant $G$ (and from $\hbar$ and $c$ which we have put to 1 ) is the highest energy scale relevant to particle physics. On the other hand, ordinary matter receives almost all of its mass from protons and neutrons which have a mass $M \approx 1 \mathrm{GeV}$. Can we understand why nucleons exist so far below the Planck scale? This is a typical hierarchy problem of which there are several in physics (also including the notorious cosmological constant problem). As Wilczek has pointed out, the large hierarchy between the Planck scale $M_{P}$ and the nucleon mass $M$ is responsible for the feebleness of gravity [1]. To understand this, let us compare the strengths of the gravitational attraction and the electrostatic repulsion between two protons at some large distance $R$. The force of gravity is given by

$$
F_{g}=G \frac{M^{2}}{R^{2}}
$$

while the electrostatic force is

$$
F_{e}=\frac{e^{2}}{R^{2}}
$$

where $e$ is the proton's electric charge. The ratio of the two forces is thus

$$
\frac{F_{g}}{F_{e}}=G \frac{M^{2}}{e^{2}} \approx 137 \frac{M^{2}}{M_{P}^{2}} \approx 10^{-36} .
$$

Hence, if we can understand why $M \ll M_{P}$, we can understand why gravity is a very weak force.

As Wilczek has explained in [1], the nucleon mass $M$ is much smaller than the Planck scale $M_{P}$ partly due to the asymptotic freedom of QCD. At the classical level, QCD with massless quarks has no dimensionful parameter at all. When the theory is quantized, a scale enters through the mechanism of dimensional transmutation. A non-zero nucleon mass arises even in massless QCD due to the confinement of colored quarks and gluons inside color-neutral hadrons. Thus, the nucleon mass $M$ is a nonperturbatively generated scale which cannot be understood using perturbation theory. The continuum theory, i.e. dimensional regularization and renormalization applied to the QCD Lagrangian, is not even defined at a nonperturbative level. The only nonperturbative definition of QCD is provided by lattice field theory in which continuous space-time is replaced by a hypercubic lattice [2, [3]. In particular, it should be pointed out that lattice QCD is not an approximation to any pre-existing nonperturbatively well-defined theory in the continuum. Of course, as in any other quantum field theory, one must ultimately remove the cut-off. On the lattice, the shortest physical distance is the lattice spacing $a$ which defines an ultraviolet momentum cut-off $1 / a$. Removing the cut-off thus means taking the continuum limit $a \rightarrow 0$. The masses of hadrons $M=1 / \xi$ are the inverse of a correlation length $\xi$. Taking the continuum limit means that the physical mass $M$ must be much smaller than the cut-off, i.e.

$$
M \ll \frac{1}{a} \Rightarrow \xi \gg a \text {. }
$$

Hence, in the continuum limit the physical correlation length $\xi$ goes to infinity in units of the lattice spacing. In the language of classical statistical mechanics this corresponds to a second order phase transition.

Most of the time, lattice QCD is used as a very powerful tool for numerical calculations of hadronic properties. However, the lattice can do more than that. To illustrate this, we will now use lattice 
QCD to explain why nucleons can exist naturally far below the Planck scale. Of course, it is wellknown that QCD is not valid up to the Planck scale. In particular, it is embedded in the standard model which itself is an effective theory limited to energies below about $1 \mathrm{TeV}$. However, unlike the full standard model, thanks to asymptotic freedom QCD alone makes sense at arbitrarily high energy scales. Whatever replaces QCD and the standard model at ultra-short distances — be it string theory or some tiny wheels turning around at the Planck scale - Nature must have found a concrete way to regularize the QCD physics at ultra-short distances. Due to renormalizability and universality, only the symmetries but not the details of this regularization should matter at low energies. For simplicity, we will use lattice QCD (and not, for example, string theory) as an admittedly oversimplified model of Nature at ultra-short distances. In other words, in this context we like to identify the lattice cut-off $1 / a$ with the Planck scale $M_{P}$.

Using lattice QCD, how can we then understand why the nucleon mass $M$ is far below $M_{P}=1 / a$ ? As Wilczek pointed out, one key ingredient is asymptotic freedom. Thanks to asymptotic freedom, without any fine-tuning of the bare gauge coupling a non-Abelian lattice Yang-Mills theory produces a correlation length $\xi$ that is larger than the lattice spacing $a$ by a factor exponentially large in the inverse coupling. In particular, choosing a bare coupling that is not unnaturally small, one can easily generate a hierarchy of scales like $M_{P} / M=\xi / a \approx 10^{19}$. Interestingly, the situation is not as simple when one proceeds from a pure gluon Yang-Mills theory to full lattice QCD including quarks. In particular, unlike continuum QCD, lattice QCD does not naturally have a chiral symmetry that can protect the quark masses from running up to the lattice "Planck scale" 1/a. Indeed, for about two decades lattice field theorists have suffered from a hierarchy problem in the fermion sector. This problem first arose when Wilson removed the unwanted doubler fermions by breaking chiral symmetry explicitly [3]. Recovering chiral symmetry in the continuum limit then requires a delicate fine-tuning of the bare fermion mass. In particular, if at ultra-short distances Nature would be a lattice gauge theory with Wilson fermions, without unnatural fine-tuning quarks would have masses at the Planck scale and the lightest particles would be glueballs. In that case it would be very puzzling why ordinary matter consists not just of gluons, but also of light quarks. If one works in continuum QCD one often takes chiral symmetry for granted, and one may view this hierarchy puzzle just as a problem of the lattice formulation. However, one should not forget that continuum QCD is not even defined beyond perturbation theory. In addition, subtleties of the definition of $\gamma_{5}$ in the framework of dimensional regularization affect even the continuum theory, and are just another aspect of the same deep problem of chiral symmetry that is manifest on the lattice. Indeed, there is a severe hierarchy problem for nonperturbative fermion dynamics that Nature must have solved somehow because it presents us with nucleons that exist far below the Planck scale.

Remarkably, the long-standing hierarchy problem of lattice fermions - and hence of the nonperturbative regularization of chiral symmetry — has recently been solved very elegantly. Using previous research of Callan and Harvey [4], Kaplan [5] realized that massless four-dimensional lattice fermions arise naturally, i.e. without fine-tuning, as zero-modes localized on a domain wall embedded in a fivedimensional space-time. In particular, left- and right-handed fermions can be localized on a domain wall and an anti-wall. When the wall and the anti-wall are separated by a sufficiently large distance, the left- and right-handed modes cannot mix, simply because they are spatially separated. As a result, a Dirac fermion arises which is protected from picking up a large mass and which is thus naturally light. Remarkably, in contrast to four dimensions, a Wilson term in a five-dimensional lattice theory removes the doubler fermions without breaking the chiral symmetry of the light four-dimensional domain wall fermions.

When Kaplan proposed his idea of regulating chiral fermions using domain walls, Narayanan and Neuberger were developing independently another approach to regulating chiral fermions using an infinite number of flavors [6]. Based on this approach they developed what is now referred to as overlap lattice fermions [7, 8. Since the flavor-space can be viewed as an extra dimension, the overlap approach is closely related to the domain wall approach. When one separates the wall and the anti-wall by an 
infinite distance, domain wall fermions turn into overlap fermions. Overlap fermions have the advantage that they have an exact chiral symmetry, while the chiral symmetry of domain wall fermions is only approximate for a finite wall-anti-wall separation. Both overlap and domain wall fermions yield naturally light quarks, and both are naturally related to the physics of a higher-dimensional space-time.

Hasenfratz and Niedermayer have investigated nonperturbative renormalization group blocking transformations on the lattice [9]. The fixed points of such transformations correspond to lattice actions which are completely free of cut-off effects - so-called perfect actions. Perfect actions for pure gauge theory, as well as for free Wilson and staggered fermions were investigated in [10, 11, 12, 13. Classically perfect actions for full QCD have been constructed in [14, 15. In the process of these investigations Hasenfratz (see [16]) rediscovered an old paper by Ginsparg and Wilson [17. He also realized that what is now called the Ginsparg-Wilson relation is the key to understanding chiral symmetry on the lattice. The Ginsparg-Wilson relation represents a general requirement on a lattice action which guarantees that it has good chiral properties. When Ginsparg and Wilson discovered this relation, it seemed impossible to explicitly construct lattice actions that obey it. By now it has been shown that classically perfect lattice actions can be approximated well enough, so that the Ginsparg-Wilson action is satisfied with high accuracy. From the point of view of practical lattice QCD calculations this represents very important progress. However, the explicit construction of perfect actions is a delicate problem that can be considered a very elaborate form of fine-tuning. Hence, it seems unnatural that Nature has chosen anything like a perfect action to regularize the strong interactions at ultra-short distances. Unlike perfect fermions, overlap fermions can describe massless quarks in QCD without fine-tuning. By integrating out the extra dimension, Neuberger has constructed lattice Dirac operators for massless quarks analytically [18, 19] and these Dirac operators do indeed satisfy the Ginsparg-Wilson relation exactly 20. Remarkably, both overlap as well as domain wall fermions, which naturally have a chiral symmetry without fine-tuning, are related to the physics in a higher-dimensional space-time. Hence, the existence of light four-dimensional fermions may be a concrete hint to the physical reality of extra dimensions.

The full strength of the Ginsparg-Wilson relation was realized by Lüscher who discovered that it suggests a natural definition of lattice chiral symmetry which reduces to the usual one in the continuum limit 21]. Based on this insight, Lüscher achieved a spectacular breakthrough: the nonperturbative construction of lattice chiral gauge theories [22]. Hence, not only QCD in which chiral symmetry is global, but also the standard model with its local chiral symmetry now stands on a solid nonperturbative basis. Even continuum perturbation theory can benefit from these developments. In particular, the ambiguities in the definition of $\gamma_{5}$ that arise in multi-loop calculations using dimensional regularization can be eliminated when one uses the lattice regularization. Still, there is a very long way to go from Lüscher's theoretical construction to practical numerical calculations in chiral gauge theories like the standard model.

The situation is a lot simpler, but still highly nontrivial, in applications of Ginsparg-Wilson fermions to simulations of QCD. Compared to the standard Wilson or staggered lattice fermions, which are already very difficult to treat fully dynamically, domain wall, overlap, or perfect fermions demand even much larger computing power. Hence, at present they are often used in the quenched approximation in which the fermion determinant is ignored. If one does not want to wait a long time for even bigger computers, it will require an algorithmic breakthrough to bring the theoretical developments of lattice chiral symmetry to fruition in fully dynamical simulations of lattice QCD. Promising steps in this direction are already being taken [23, 24].

If one imagines that Nature has used something like domain wall fermions to regularize the strong interactions, it is natural that nucleons (and not just glueballs) exist far below the Planck scale. However, it remains mysterious where the quark masses themselves come from. In the standard model the quark masses arise from Yukawa couplings to the Higgs field, but the values of these couplings are free parameters. Still, within the standard model the traditional gauge hierarchy problem arises: why is the 
electroweak scale so small compared to the Planck scale? Chiral symmetry can protect fermion masses from running to the ultimate high-energy cut-off, but it cannot protect scalars. A potential solution of the gauge hierarchy problem is provided by supersymmetry. Supersymmetry relates scalars to fermions and thus allows chiral symmetry to indirectly generate naturally light scalars as well. At a nonperturbative level, supersymmetry is as undefined as chiral symmetry was before the recent developments on the lattice. In the worst case, supersymmetry may just be a perturbative illusion which does not arise naturally at a nonperturbative level. Unfortunately, unlike for chiral symmetry, Nature has not yet provided us with experimental evidence for supersymmetry (except as an accidental symmetry in heavy nuclei). Hence, one cannot be sure that it is indeed possible to construct naturally light scalars at a nonperturbative level. Perhaps the many beautiful results obtained within supersymmetric continuum theories should make us optimistic that these theories actually exist at a rigorous level beyond perturbation theory. Again, Kaplan and his collaborators have taken very interesting steps towards constructing supersymmetric theories on the lattice [25]. It remains to be seen if these developments will lead to a repetition of the Ginsparg-Wilson revolution of lattice chiral symmetry.

This review is an introduction to chiral symmetry on the lattice. We assume that the reader has a background in continuum field theory but not necessarily in lattice field theory. It should be noted that there are very good textbooks that cover the basics of lattice field theory much more completely than it can be done here [26, 27, 28. Furthermore, there are excellent reviews of the more advanced and specialized aspects of lattice field theory and chiral symmetry [29, 30, 31, 32. This review aims at bridging some of the gaps between the basic and the more advanced texts and introducing the reader to some of the latest developments in the field. In section 2 we summarize symmetry properties of QCD in the continuum. We concentrate on chiral symmetry, but also discuss scale invariance, the axial anomaly, and the Atiyah-Singer index theorem. Section 3 illustrates basic properties of lattice chiral symmetry using free fermions. In particular, we discuss the fermion doubling problem, the Nielsen-Ninomiya theorem, and Wilson, staggered, as well as perfect fermions. In section 4, gluons are added and full lattice QCD is considered. In particular, the Ginsparg-Wilson relation is discussed. Section 5 concerns special features of Ginsparg-Wilson fermions, including the axial anomaly and the index theorem on the lattice, the Witten-Veneziano mass formula, the renormalization of operators, as well as numerical simulations of Ginsparg-Wilson fermions. Section 6 discusses effective theories for pions, nucleons, and constituent quarks, both in the continuum and on the lattice. In particular, the nonlinear realization of chiral symmetry implies another way of solving the fermion doubling problem. Lattice simulations of constituent quarks may eventually shed some light on the success of the nonrelativistic quark model. Finally, section 7 contains our conclusions.

\section{Symmetries of the Strong Interactions}

In this section we review some aspects of symmetries in the continuum formulation of QCD with an emphasis on chiral symmetry.

\subsection{SU $\left(N_{c}\right)$ Yang-Mills Theory in the Continuum}

Let us consider an anti-Hermitean non-Abelian $S U\left(N_{c}\right)$ gauge field

$$
A_{\mu}(x)=i g A_{\mu}^{a}(x) T^{a}
$$

which (for $N_{c}=3$ ) describes the gluons of QCD. Here $g$ is the gauge coupling, $A_{\mu}^{a}(x)$ (with $a \in$ $\left.\left\{1,2, \ldots, N_{c}^{2}-1\right\}\right)$ is the real-valued non-Abelian vector potential at the Euclidean space-time point $x$, and the $T^{a}$ (which obey $\left.\operatorname{Tr}\left(T^{a} T^{b}\right)=\frac{1}{2} \delta_{a b}\right)$ are the Hermitean generators of the $S U\left(N_{c}\right)$ algebra. The 
algebra-valued field strength takes the form

$$
F_{\mu \nu}(x)=\partial_{\mu} A_{\nu}(x)-\partial_{\nu} A_{\mu}(x)+\left[A_{\mu}(x), A_{\nu}(x)\right]
$$

and the corresponding Euclidean Yang-Mills action is given by

$$
S_{Y M}[A]=-\int d^{4} x \frac{1}{2 g^{2}} \operatorname{Tr}\left(F_{\mu \nu} F_{\mu \nu}\right)
$$

The action is invariant under group-valued gauge transformations $\Omega(x) \in S U\left(N_{c}\right)$,

$$
A_{\mu}^{\prime}(x)=\Omega(x)\left(A_{\mu}(x)+\partial_{\mu}\right) \Omega(x)^{\dagger},
$$

under which the field strength transforms as

$$
F_{\mu \nu}(x)=\Omega(x) F_{\mu \nu}(x) \Omega(x)^{\dagger} .
$$

The quantum theory is defined by a functional integral over all gluon fields

$$
Z=\int \mathcal{D} A \exp \left(-S_{Y M}[A]\right)
$$

which is a formal expression before it is properly regularized. In perturbation theory this is possible using standard dimensional regularization techniques. Through the regularization, a scale is introduced into the quantum theory which explicitly breaks the scale invariance of the classical Yang-Mills theory. This anomaly in the scale invariance is responsible for the phenomenon of dimensional transmutation: in the quantum theory the dimensionless coupling constant $g$ of the classical theory is traded for a dimensionful scale. In the modified minimal subtraction renormalization scheme this scale is $\Lambda_{\overline{M S}}$ which is defined in the framework of perturbation theory. We will soon define the theory beyond perturbation theory by regularizing it on a space-time lattice. In a nonperturbative context, a natural scale is the dynamically generated mass gap $M$ - the energy of the lowest state above the vacuum. In a Yang-Mills theory this state is the lightest glueball. The $S U\left(N_{c}\right)$ Yang-Mills theory is a quantum theory without any free parameter. For example, the dimensionless ratio $M / \Lambda_{\overline{M S}}$ is a pure number predicted by the theory. The relation of $M$ or $\Lambda_{\overline{M S}}$ to units like $\mathrm{GeV}$, on the other hand, is, of course, not predicted by the theory. Such man-made mass units are related to the kilogram, defined by the arbitrary amount of platinum-iridium alloy deposited near Paris a long time ago.

Another quantity of physical interest is the topological charge

$$
Q[A]=-\frac{1}{32 \pi^{2}} \int d^{4} x \varepsilon_{\mu \nu \rho \sigma} \operatorname{Tr}\left(F_{\mu \nu} F_{\rho \sigma}\right) \in \Pi_{3}\left[S U\left(N_{c}\right)\right]=\mathbf{Z}
$$

which takes integer values in the third homotopy group of the gauge group. The topological charge gives rise to an additional parameter, the vacuum angle $\theta$, in the Yang-Mills functional integral

$$
Z(\theta)=\int \mathcal{D} A \exp \left(-S_{Y M}[A]+i \theta Q[A]\right)
$$

For $\theta \neq 0$ or $\pi$ the $\theta$-term explicitly breaks parity as well as CP. The bound $|\theta|<10^{-9}$ derived from the measurement of the electric dipole moment of the neutron suggests that $\theta=0$ in Nature. This result is puzzling because in the Standard Model CP is already explicitly broken by the complex phase of the Cabbibo-Kobayashi-Maskawa matrix. The puzzle to understand why $\theta=0$ is known as the strong CP problem. 


\subsection{QCD with $N_{f}$ Quark Flavors}

In the next step we add $N_{f}$ massless quarks to the pure gluon theory. Quarks and anti-quarks are described by anti-commuting Dirac spinor fields $\psi(x)$ and $\bar{\psi}(x)$. In Euclidean space-time these two fields represent independent Grassmann degrees of freedom. Under a non-Abelian gauge transformation the quark and anti-quark fields transform in the fundamental representations $\left\{N_{c}\right\}$ and $\left\{\overline{N_{c}}\right\}$, respectively, i.e.

$$
\psi(x)^{\prime}=\Omega(x) \psi(x), \bar{\psi}(x)^{\prime}=\bar{\psi}(x) \Omega(x)^{\dagger} .
$$

The fermionic part of the Euclidean action of massless QCD takes the form

$$
S_{F}[\bar{\psi}, \psi, A]=\int d^{4} x \bar{\psi} \gamma_{\mu}\left(\partial_{\mu}+A_{\mu}\right) \psi
$$

which is gauge invariant by construction. The Euclidean Dirac matrices are Hermitean and obey the anti-commutation relations

$$
\left\{\gamma_{\mu}, \gamma_{\nu}\right\}=2 \delta_{\mu \nu},\left\{\gamma_{\mu}, \gamma_{5}\right\}=0, \gamma_{5}=\gamma_{1} \gamma_{2} \gamma_{3} \gamma_{4}
$$

We now decompose the quark fields into left- and right-handed components

$$
\begin{aligned}
& \psi_{L}(x)=P_{L} \psi(x), \psi_{R}(x)=P_{R} \psi(x), \psi(x)=\psi_{L}(x)+\psi_{R}(x), \\
& \bar{\psi}_{L}(x)=\bar{\psi}(x) P_{R}, \bar{\psi}_{R}(x)=\bar{\psi}(x) P_{L}, \bar{\psi}(x)=\bar{\psi}_{L}(x)+\bar{\psi}_{R}(x) .
\end{aligned}
$$

The chiral projectors are given by

$$
P_{R}=\frac{1+\gamma_{5}}{2}, P_{L}=\frac{1-\gamma_{5}}{2}
$$

Inserting the decomposed spinors into the fermionic part of the action one obtains

$$
S_{F}[\bar{\psi}, \psi, A]=\int d^{4} x\left[\bar{\psi}_{L} \gamma_{\mu}\left(\partial_{\mu}+A_{\mu}\right) \psi_{L}+\bar{\psi}_{R} \gamma_{\mu}\left(\partial_{\mu}+A_{\mu}\right) \psi_{R}\right]
$$

i.e. the action decouples into two contributions from left- and right-handed quarks.

As a result, the action of massless QCD is invariant against $U\left(N_{f}\right)_{L} \otimes U\left(N_{f}\right)_{R}$ chiral transformations

$$
\begin{aligned}
& \psi_{L}^{\prime}(x)=L \psi_{L}(x), \bar{\psi}^{\prime}(x)=\bar{\psi}_{L}(x) L^{+}, L \in U\left(N_{f}\right)_{L}, \\
& \psi_{R}^{\prime}(x)=R \psi_{R}(x), \bar{\psi}^{\prime}(x)=\bar{\psi}_{R}(x) R^{+}, R \in U\left(N_{f}\right)_{R} .
\end{aligned}
$$

Due to an anomaly in the axial $U(1)_{A}$ symmetry, the symmetry of the quantum theory is reduced to $S U\left(N_{f}\right)_{L} \otimes S U\left(N_{f}\right)_{R} \otimes U(1)_{B}$ where the $U(1)_{B}=U(1)_{L=R}$ symmetry represents baryon number conservation.

Chiral symmetry is only approximate in Nature, because the quark mass terms couple left- and right-handed fermions. The mass terms in the QCD action take the form

$$
S_{M}[\bar{\psi}, \psi]=\int d^{4} x\left[\bar{\psi}_{R} \mathcal{M} \psi_{L}+\bar{\psi}_{L} \mathcal{M}^{\dagger} \psi_{R}\right]
$$

which is again gauge invariant but no longer chirally invariant. The quark mass matrix takes the form

$$
\mathcal{M}=\operatorname{diag}\left(m_{u}, m_{d}, m_{s}, \ldots, m_{N_{f}}\right) \text {. }
$$

If all quark masses are equal, i.e. if $\mathcal{M}=m \mathbb{1}$, the mass term is invariant only against simultaneous transformations $L=R$. Hence, chiral symmetry is then explicitly broken down to

$$
S U\left(N_{f}\right)_{L=R} \otimes U(1)_{L=R}=S U\left(N_{f}\right)_{F} \otimes U(1)_{B}
$$


which corresponds to the flavor and baryon number symmetry. In Nature the quark masses are all different, and the symmetry is, in fact, explicitly broken down to

$$
\prod_{f=1}^{N_{f}} U(1)_{f}=U(1)_{u} \otimes U(1)_{d} \otimes U(1)_{s} \otimes \ldots \otimes U(1)_{N_{f}} .
$$

The physical up and down quark masses are a lot smaller than $\Lambda_{\overline{M S}}$ while the strange quark mass is of the order of $\Lambda_{\overline{M S}}$. Consequently, $S U(2)_{L} \otimes S U(2)_{R}$ is a very good approximate global symmetry, while $S U(3)_{L} \otimes S U(3)_{R}$ is broken more strongly. It should be noted that the actual values of the quark masses are reasonably well-known from comparison with experiment, but are at present not at all understood theoretically. In particular, we don't know why there are three light quark flavors. Before one understands the relevant physics beyond the Standard Model, the origin of the chiral symmetry of QCD remains mysterious and the symmetry itself seems accidental.

The total action of QCD is simply given by

$$
S_{Q C D}[\bar{\psi}, \psi, A]=S_{Y M}[A]+S_{F}[\bar{\psi}, \psi, A]+S_{M}[\bar{\psi}, \psi]
$$

and the corresponding QCD functional integral is

$$
Z=\int \mathcal{D} \bar{\psi} \mathcal{D} \psi \mathcal{D} A \exp \left(-S_{Q C D}[\bar{\psi}, \psi, A]\right)
$$

Again, this is a formal mathematical expression before it is properly regularized. In the continuum this can be done only perturbatively. We will soon discuss the lattice regularization which defines QCD beyond perturbation theory.

\subsection{The Axial Anomaly and the Atiyah-Singer Index Theorem}

The $U(1)_{A}$ symmetry of the classical action of massless QCD is explicitly broken by quantum effects. As a consequence of this anomaly the flavor-singlet axial current

$$
j_{\mu}^{5}(x)=\bar{\psi}(x) \gamma_{\mu} \gamma_{5} \psi(x)
$$

which is conserved at the classical level, has a non-zero divergence

$$
\partial_{\mu} j_{\mu}^{5}(x)=-\frac{N_{f}}{32 \pi^{2}} \varepsilon_{\mu \nu \rho \sigma} \operatorname{Tr}\left[F_{\mu \nu}(x) F_{\rho \sigma}(x)\right]
$$

due to instantons (and other topological charge carriers) in the quantum theory. In particular, the variation of the axial charge $Q^{5}(t)=\int d^{3} x j_{0}(\vec{x}, t)$ is given by

$$
Q^{5}(t=\infty)-Q^{5}(t=-\infty)=N_{f} Q[A]
$$

where $Q[A]$ is the topological charge of eq.(12). Only in the $N_{c} \rightarrow \infty$ limit the anomaly vanishes and the chiral symmetry of massless QCD is enhanced to the full $U\left(N_{f}\right)_{L} \otimes U\left(N_{f}\right)_{R}$ group.

The axial anomaly is deeply connected with the Atiyah-Singer index theorem, which relates the zero-modes of the massless $\left(N_{f}\right.$-flavor) Dirac operator $D[A]=\gamma_{\mu}\left(\partial_{\mu}+A_{\mu}\right)$ to the topological charge $Q[A]$. The eigenvalues of the Dirac operator are purely imaginary and come in complex conjugate pairs. Only the zero eigenvalues are not paired. Since the Dirac operator anti-commutes with $\gamma_{5}$, the eigenvectors of the zero-modes (which obey $D[A] \psi=0$ ) have a definite handedness, i.e. $\gamma_{5} \psi= \pm \psi$. The index theorem states that

$$
\operatorname{index}(D[A])=n_{-}-n_{+}=N_{f} Q[A]
$$


i.e. the index of the operator $D[A]$, which is defined as the difference between the number of left- and right-handed zero-modes, is given by the topological charge.

As a consequence of the index theorem, topologically nontrivial gluon field configurations (with $Q[A] \neq 0)$ necessarily induce zero-modes in the Dirac operator and thus lead to a vanishing fermion determinant $\operatorname{det} D[A]=0$. As a function of the vacuum angle $\theta$, the functional integral of massless QCD takes the form

$$
\begin{aligned}
Z(\theta) & =\int \mathcal{D} \bar{\psi} \mathcal{D} \psi \mathcal{D} A \exp \left(-S_{Q C D}[\bar{\psi}, \psi, A]+i \theta Q[A]\right) \\
& =\int \mathcal{D} A \operatorname{det} D[A] \exp \left(-S_{Y M}[A]+i \theta Q[A]\right) \\
& =\int \mathcal{D} A \operatorname{det} D[A] \exp \left(-S_{Y M}[A]\right)=Z(0)
\end{aligned}
$$

Since $\operatorname{det} D[A]=0$ when $Q[A] \neq 0$, there are no $\theta$-vacuum effects in massless QCD. This would "solve" the strong CP problem (why is $\theta=0$ ?) if, for example, the up quark would be massless. Of course, this would leave us with the "up quark problem": why should $m_{u}=0$ ? In any case, $m_{u}=0$ seems not to be realized in Nature and the strong CP problem remains puzzling.

\subsection{Spontaneous Chiral Symmetry Breaking}

Due to the approximate chiral symmetry of QCD one would expect corresponding near degeneracies in the spectrum of strongly interacting particles. Indeed, hadrons can be classified as isospin multiplets. The isospin transformations act on left- and right-handed fermions simultaneously, i.e. $S U(2)_{I}=S U(2)_{L=R}$. The $S U(3)_{F}=S U(3)_{L=R}$ flavor symmetry is more approximate but is still clearly visible in the spectrum. The full $S U\left(N_{f}\right)_{L} \otimes S U\left(N_{f}\right)_{R} \otimes U(1)_{B}$ chiral symmetry, on the other hand, is not manifest in the spectrum at all. In particular, one does not observe mass-degenerate parity doublets of hadrons, as one should if chiral symmetry was manifest in the spectrum. Furthermore, one observes very light pseudo-scalar particles — the pions $\pi^{+}, \pi^{0}$, and $\pi^{-}$- as well as somewhat heavier pseudo-scalars - the four kaons $K^{+}, K^{0}, \overline{K^{0}}, K^{-}$and the $\eta$-meson.

¿From the experimental evidence one concludes that chiral symmetry must be spontaneously broken. Indeed, when a continuous global symmetry breaks spontaneously, massless Goldstone bosons appear

in the spectrum. According to Goldstone's theorem, the number of massless bosons is given by the difference of the number of generators of the full symmetry group $G$ and the subgroup $H$ that remains unbroken. In massless QCD the full chiral symmetry group is

$$
G=S U\left(N_{f}\right)_{L} \otimes S U\left(N_{f}\right)_{R} \otimes U(1)_{B}
$$

while the unbroken subgroup is the flavor symmetry

$$
H=S U\left(N_{f}\right)_{L=R} \otimes U(1)_{B}
$$

Hence, in this case one expects $N_{f}^{2}-1$ massless Goldstone bosons. For $N_{f}=2$ these are the three pions, while for $N_{f}=3$ there are eight Goldstone bosons - the pions, the kaons, and the $\eta$-meson. In Nature these particles are not exactly massless, because chiral symmetry is explicitly broken by the quark masses. The masses of the up and down quarks are much smaller than the QCD scale $\Lambda_{\overline{M S}}$ which leads to the very small pion mass. The mass of the strange quark, on the other hand, is of the order of $\Lambda_{\overline{M S}}$, thus leading to larger masses of the kaons and the $\eta$-meson. Still, their masses are small enough to identify these particles as pseudo-Goldstone bosons.

Chiral symmetry breaking has not yet been derived analytically from the QCD Lagrangian. In particular, spontaneous chiral symmetry breaking is a nonperturbative phenomenon whose understanding 
requires a formulation of QCD beyond perturbation theory. Such a formulation is provided by lattice field theory which will be discussed below. In the lattice formulation, in the strong coupling limit it is possible to show rigorously that chiral symmetry is indeed spontaneously broken 33, 34. Further, numerical simulations in lattice QCD confirm that chiral symmetry is spontaneously broken even at weaker couplings. For example, one detects spontaneous chiral symmetry breaking by investigating the chiral order parameter

$$
\langle\bar{\psi} \psi\rangle=\langle 0|\bar{\psi}(x) \psi(x)| 0\rangle=\left\langle 0\left|\bar{\psi}_{R}(x) \psi_{L}(x)+\bar{\psi}_{L}(x) \psi_{R}(x)\right| 0\right\rangle .
$$

The order parameter is invariant against simultaneous transformations $R=L$, but not against general chiral rotations. If chiral symmetry would be intact the chiral condensate would vanish. When the symmetry is spontaneously broken, on the other hand, $\langle\bar{\psi} \psi\rangle$ is non-zero.

\section{$3 \quad$ Free Lattice Fermions}

In this section we begin to formulate QCD on a space-time lattice which serves as an ultraviolet regulator. We replace Euclidean space-time by a hypercubic lattice of points $x$ with lattice spacing $a$. The lattice provides an ultraviolet momentum cut-off $1 / a$. The continuum limit is reached when $a \rightarrow 0$. One has a lot of freedom in writing down a lattice regularized theory. In order to ensure that one reaches the desired theory in the continuum limit one must pay attention to the relevant symmetries. The most important symmetry of QCD is the $S U\left(N_{c}\right)$ gauge invariance. It is an important strength of the lattice regularization that it manifestly respects gauge invariance. The fact that space-time symmetries are explicitly broken down to discrete translations and the hypercubic rotation group of the lattice is not a severe problem. In particular, in QCD the hypercubic symmetry is powerful enough to ensure that the full Poincaré symmetry of the continuum is automatically recovered as $a \rightarrow 0$. Discrete symmetries like parity and charge conjugation are also easy to maintain on the lattice. This review concentrates on the question of how to realize chiral symmetry on the lattice. In this section we consider lattice theories of free quarks only. Gluon fields will be added in the next section.

\subsection{The Naive Lattice Fermion Action and the Doubling Problem}

In the continuum the Euclidean action of a free Dirac fermion in $d$ space-time dimensions is given by

$$
S[\bar{\psi}, \psi]=\int d^{d} x \bar{\psi}\left(\gamma_{\mu} \partial_{\mu}+m\right) \psi
$$

and the functional integral takes the form

$$
Z=\int \mathcal{D} \bar{\psi} \mathcal{D} \psi \exp (-S[\bar{\psi}, \psi])
$$

On the lattice the continuum fermion field $\bar{\psi}(x), \psi(x)$ is replaced by Grassmann variables $\bar{\Psi}_{x}, \Psi_{x}$ which live on the lattice points $x$. The continuum derivative can be discretized by a finite difference, such that

$$
S[\bar{\Psi}, \Psi]=a^{d} \bar{\Psi} D \Psi=a^{d} \sum_{x, \mu} \frac{1}{2 a}\left(\bar{\Psi}_{x} \gamma_{\mu} \Psi_{x+\hat{\mu}}-\bar{\Psi}_{x+\hat{\mu}} \gamma_{\mu} \Psi_{x}\right)+a^{d} \sum_{x} m \bar{\Psi}_{x} \Psi_{x}
$$

Here $\hat{\mu}$ is a vector of length $a$ in the $\mu$-direction. In the continuum limit $a \rightarrow 0$ the lattice sum $a^{d} \sum_{x}$ becomes the continuum integral $\int d^{d} x$ over space-time. The corresponding lattice Dirac operator which is a matrix in the Dirac- and space-time indices takes the form

$$
D_{x, y}=\sum_{\mu} \frac{1}{2 a}\left(\gamma_{\mu} \delta_{x+\hat{\mu}, y}-\gamma_{\mu} \delta_{x-\hat{\mu}, y}\right)+m \delta_{x, y}
$$


The lattice functional integral can be written as

$$
Z=\int \mathcal{D} \bar{\Psi} \mathcal{D} \Psi \exp \left(-a^{d} \bar{\Psi} D \Psi\right)=\prod_{x} \int d \bar{\Psi}_{x} d \Psi_{x} \exp (-S[\bar{\Psi}, \Psi])
$$

In particular, the fermionic Grassmann integration measure is completely regularized explicitly.

The momentum space of the lattice theory is a $d$-dimensional Brillouin zone $B=[-\pi / a, \pi / a]^{d}$ with periodic boundary conditions. Going to momentum space, the naive fermion action from above gives rise to the lattice fermion propagator

$$
\langle\bar{\Psi}(-p) \Psi(p)\rangle=\left[i \sum_{\mu} \gamma_{\mu} \frac{1}{a} \sin \left(p_{\mu} a\right)+m\right]^{-1} .
$$

By performing a Fourier transform in the Euclidean energy $p_{d}$ one obtains the fermion 2-point function

$$
\left\langle\bar{\Psi}(-\vec{p}, 0) \Psi\left(\vec{p}, x_{d}\right)\right\rangle=\frac{1}{2 \pi} \int_{-\pi / a}^{\pi / a} d p_{d}\langle\bar{\Psi}(-p) \Psi(p)\rangle \exp \left(i p_{d} x_{d}\right) \sim \exp \left(-E(\vec{p}) x_{d}\right) .
$$

At large Euclidean time separation $x_{d}$ the 2-point function decays exponentially with the energy $E(\vec{p})$ of a fermion with spatial momentum $\vec{p}$. For the naive fermion action the lattice dispersion relation takes the form

$$
\sinh ^{2}(E(\vec{p}) a)=\sum_{i} \sin ^{2}\left(p_{i} a\right)+(m a)^{2} .
$$

The continuum dispersion relation $E(\vec{p})^{2}=\vec{p}^{2}+m^{2}$ is indeed recovered in the continuum limit $a \rightarrow 0$. However, besides $\vec{p}=0$ there are other momenta $\vec{p}$ for which $E(\vec{p})$ becomes small. These are located at the corners of the Brillouin zone where the components of the momentum vector take the values $p_{i}=0$ or $\pi / a$, such that $\sin \left(p_{i} a\right)=0$. As a consequence, the lattice dispersion relation leads to additional states in the spectrum which are absent in the continuum theory and which do not disappear in the continuum limit. Hence, the naive lattice fermion action does not lead to the correct continuum theory. The extra states appearing in the lattice dispersion relation show up as additional physical particles - the so-called doubler fermions. Fermion doubling is a manifestation of a deep fundamental problem of lattice regularized fermionic theories with a chiral symmetry. The fermion doubling problem leads to a multiplication of fermion species. The lattice fermion propagator of eq.(40) has $2^{d}$ poles instead of just one as in the continuum. The origin of the doubling problem is deeply connected with chiral symmetry and can be traced back to the axial anomaly. The doubler fermions pose a severe problem in lattice field theory. Without removing them we cannot describe Nature's QCD (which has 3 and not $2^{d}=2^{4}=16$ light quark flavors).

\subsection{The Nielsen-Ninomiya Theorem}

Before we try to eliminate the doubler fermions let us prove a general theorem due to Nielsen and Ninomiya 35]: a chirally invariant free fermion lattice action, which is local, translation invariant, and real necessarily has fermion doubling. The theorem is based on topology. It holds because the lattice momentum space (the Brillouin zone $B$ ) is a torus. A general chirally symmetric and translationally invariant lattice action for free fermions takes the form

$$
S[\bar{\Psi}, \Psi]=a^{d} \sum_{x, y} \bar{\Psi}_{x} \gamma_{\mu} \rho_{\mu}(x-y) \Psi_{y}
$$

The function $\rho_{\mu}(x-y)$ determines the strength of the coupling between the fermion field values $\bar{\Psi}_{x}$ and $\Psi_{y}$ at two points $x$ and $y$ which may be separated by an arbitrarily large distance. Locality of the lattice action does not mean that the points $x$ and $y$ must be nearest neighbors. It only means 
that $\rho_{\mu}(x-y)$ decays exponentially at large separations $x-y$. Going to momentum space, locality implies that in Fourier space $\rho_{\mu}(p)$ is a regular function (without poles) over the Brillouin zone. The corresponding lattice fermion propagator takes the form

$$
\langle\bar{\Psi}(-p) \Psi(p)\rangle=\left[i \sum_{\mu} \gamma_{\mu} \rho_{\mu}(p)\right]^{-1} .
$$

Reality and translation invariance of the lattice action imply that $\rho_{\mu}(p)$ is a real-valued periodic function over the Brillouin zone.

Poles of the propagator - and hence physical or doubler fermions - correspond to zeros of $\rho_{\mu}(p)$, i.e. to points $p$ with $\rho_{\mu}(p)=0$ for all $\mu$. The Nielsen-Ninomiya theorem states that a regular, real-valued, and periodic function $\rho_{\mu}(p)$ necessarily vanishes at more than just one point. It is trivial to prove this for $d=1$. In that case, there is a single regular periodic function $\rho_{1}(p)$ which should at least have one zero in order to describe the physical fermion pole. The function is positive on one side of the zero and negative on the other side. Hence, it must go through zero again in order to satisfy periodicity, thus leading to a doubler fermion pole in the lattice propagator. In higher dimensions the proof is analogous. For example, for $d=2$ there are two functions $\rho_{1}(p)$ and $\rho_{2}(p)$. The zeros of $\rho_{1}(p)$ lie on a closed curve in the two-dimensional Brillouin zone. This curve may be closed via the periodic boundary conditions. The zeros of $\rho_{2}(p)$ lie on another closed curve that intersects the first one in the pole position of the physical fermion. Due to the periodic boundary conditions of the Brillouin zone, the two curves must necessarily also intersect somewhere else. The curves cannot just touch each other because this would lead to an incorrect dispersion relation for the physical fermion. In $d$ dimensions the zeros of $\rho_{\mu}(p)$ (with $\mu=1,2, \ldots, d)$ lie on $d$ closed $(d-1)$-dimensional surfaces. Again, those cannot intersect in just one point. If they intersect once they necessarily intersect also somewhere else. This proves lattice fermion doubling for a chirally symmetric, translation invariant, real-valued lattice action. It should be noted that the theorem does not specify the number of doubler fermions. It is indeed possible to reduce the number of doublers from $2^{d}-1$ to 1 , but it is impossible to eliminate the doubler fermions completely.

One may try to evade the theorem by violating one of its basic assumptions. Giving up translation invariance or the reality of the action has not led to acceptable solutions of the fermion doubling problem. Giving up locality is probably the last thing one should do in field theory. For example, the early idea of SLAC fermions [36] turned out to be unacceptable for this reason.

\subsection{Wilson Fermions}

In his work on lattice gauge theory Wilson removed the doubler fermions in a direct and radical way by breaking chiral symmetry explicitly [3]. Then the Nielsen-Ninomiya theorem is evaded because the propagator contains additional terms without $\gamma_{\mu}$. The so-called Wilson term gives the fermion doublers a mass of the order of the cut-off while the physical fermion remains massless. Hence, in the continuum limit chiral symmetry is recovered in the physical sector. Wilson's modification of the naive fermion action takes the form of a discretized second derivative

$$
\begin{aligned}
S[\bar{\Psi}, \Psi] & =a^{d} \sum_{x, \mu} \frac{1}{2 a}\left(\bar{\Psi}_{x} \gamma_{\mu} \Psi_{x+\hat{\mu}}-\bar{\Psi}_{x+\hat{\mu}} \gamma_{\mu} \Psi_{x}\right)+a^{d} \sum_{x} m \bar{\Psi}_{x} \Psi_{x} \\
& +a^{d} \sum_{x, \mu} \frac{1}{2 a}\left(2 \bar{\Psi}_{x} \Psi_{x}-\bar{\Psi}_{x} \Psi_{x+\hat{\mu}}-\bar{\Psi}_{x+\hat{\mu}} \Psi_{x}\right) .
\end{aligned}
$$

Then the lattice propagator takes the form

$$
\langle\bar{\Psi}(-p) \Psi(p)\rangle=\left[i \sum_{\mu} \gamma_{\mu} \frac{1}{a} \sin \left(p_{\mu} a\right)+m+\sum_{\mu} \frac{2}{a} \sin ^{2}\left(\frac{p_{\mu} a}{2}\right)\right]^{-1} .
$$


The Wilson term acts as a momentum-dependent mass term. For small momenta it vanishes quadratically, and hence it does not affect the dispersion of the physical fermion, at least in the continuum limit. For the doubler fermions, on the other hand, the Wilson term is non-zero, and gives them a mass of the order of the cut-off $1 / a$. In the continuum limit the doubler fermions are hence eliminated from the spectrum of the theory. Unfortunately, in lattice QCD this leads to a variety of complications. In particular, recovering chiral symmetry in the continuum limit requires unnatural fine-tuning of the bare fermion mass. For more details we refer the reader to [27, 28].

\subsection{Staggered Fermions}

Staggered fermions result from naive doubled lattice fermions by so-called spin diagonalization [37, 38. 39. In four space-time dimensions the size of the Dirac matrices is $4 \times 4$. By spin diagonalization one can reduce the fermion multiplication factor $2^{d}=16$ to $16 / 4=4$. Hence, for $d=4$, staggered fermions represent 4 flavors of mass-degenerate fermions. Staggered fermions have a single 1-component pair of Grassmann variables $\bar{\chi}_{x}$ and $\chi_{x}$ per lattice point $x$. The corresponding lattice action for free staggered fermions takes the form

$$
S[\bar{\chi}, \chi]=a^{4} \sum_{x, \mu} \frac{1}{2 a}\left(\bar{\chi}_{x} \eta_{x, \mu} \chi_{x+\hat{\mu}}-\bar{\chi}_{x+\hat{\mu}} \eta_{x, \mu} \chi_{x}\right)+a^{4} \sum_{x} m \bar{\chi}_{x} \chi_{x}
$$

where

$$
\eta_{x, 1}=1, \eta_{x, 2}=(-1)^{x_{1} / a}, \eta_{x, 3}=(-1)^{\left(x_{1}+x_{2}\right) / a}, \eta_{x, 4}=(-1)^{\left(x_{1}+x_{2}+x_{3}\right) / a} .
$$

For $m=0$ the staggered fermion action has an exact $U(1)_{e} \otimes U(1)_{o}$ symmetry

$$
\begin{gathered}
\chi_{x}^{\prime}=\exp \left(i \varphi_{e}\right) \chi_{x}, \bar{\chi}_{x}^{\prime}=\bar{\chi}_{x} \exp \left(-i \varphi_{o}\right), \text { for }\left(x_{1}+x_{2}+x_{3}+x_{4}\right) / a \text { even } \\
\chi_{x}^{\prime}=\exp \left(i \varphi_{o}\right) \chi_{x}, \bar{\chi}_{x}^{\prime}=\bar{\chi} \exp \left(-i \varphi_{e}\right), \text { for }\left(x_{1}+x_{2}+x_{3}+x_{4}\right) / a \text { odd }
\end{gathered}
$$

which is a subgroup of the $S U(4)_{L} \otimes S U(4)_{R} \otimes U(1)_{B}$ chiral symmetry of the corresponding continuum theory. In the interacting theory the chiral and flavor symmetries besides $U(1)_{e} \otimes U(1)_{o}$ are explicitly broken by the staggered fermion action. These symmetries are recovered only in the continuum limit. A detailed introduction to staggered fermions and its properties can be found in textbooks like [27, 28] and review articles like 40] which cover more advanced topics.

Unlike Nature's QCD, staggered fermions have 4 flavors of the same mass. Since they have a remnant of chiral symmetry and are relatively easy to simulate numerically, staggered fermions provide a convenient framework for studies of chiral symmetry breaking at $N_{f}=4$. Some numerical studies of staggered fermions try to mimic Nature's QCD by taking roots of the fermion determinant in order to relate 4 mass-degenerate staggered fermions to physical up, down, and strange quarks. These calculations are not on completely solid grounds before one can show that locality (which may be violated at finite lattice spacing) is recovered in the continuum limit.

\subsection{Perfect Lattice Fermions}

In this subsection we relate the continuum theory of free fermions to a corresponding lattice theory by an exact renormalization group transformation. This is achieved by defining lattice fermion fields as block averages of continuum fields integrated over hypercubes [11, 12, 13. The resulting lattice theory is in all respects equivalent to the underlying continuum theory, i.e. it is completely free of lattice artifacts. For example, it has the same energy-momentum dispersion relation as the continuum theory. Even more important, it has an exact chiral symmetry (which may, however, be hidden). Lattice actions with these properties are known as perfect actions. 
Let us derive a perfect fermion action by blocking from the continuum [12. For this purpose we average the continuum fermion field $\psi(y)$ over hypercubes $c_{x}$ of size $a^{d}$ centered at the points $x$ of a $d$-dimensional Euclidean lattice

$$
\Psi_{x}=\frac{1}{a^{d}} \int_{c_{x}} d^{d} y \psi(y), \bar{\Psi}_{x}=\frac{1}{a^{d}} \int_{c_{x}} d^{d} y \bar{\psi}(y)
$$

which in momentum space corresponds to

$$
\Psi(p)=\sum_{l \in \mathbf{Z}^{d}} \psi(p+2 \pi l / a) \Pi(p+2 \pi l / a), \bar{\Psi}(-p)=\sum_{n \in \mathbf{Z}^{d}} \bar{\psi}(-p-2 \pi n / a) \Pi(p+2 \pi n / a),
$$

Note that the lattice fermion field is periodic over the Brillouin zone. The Fourier transform of the blocking kernel is given by

$$
\Pi(p)=\prod_{\mu=1}^{d} \frac{2 \sin \left(p_{\mu} a / 2\right)}{p_{\mu} a} .
$$

The lattice fermion propagator is related to the continuum propagator by

$$
\begin{aligned}
\langle\bar{\Psi}(-p) \Psi(p)\rangle & =\sum_{l \in \mathbf{Z}^{d}}\langle\bar{\psi}(-p-2 \pi l / a) \psi(p+2 \pi l / a)\rangle \Pi(p+2 \pi l / a)^{2} \\
& =\sum_{l \in \mathbf{Z}^{d}}\left[i \gamma_{\mu}\left(p_{\mu}+2 \pi l_{\mu} / a\right)+m\right]^{-1} \Pi(p+2 \pi l / a)^{2}
\end{aligned}
$$

For $m=0$ the lattice propagator corresponds to a lattice action

$$
S[\bar{\Psi}, \Psi]=a^{d} \sum_{x, y} \bar{\Psi}_{x} \gamma_{\mu} \rho_{\mu}(x-y) \Psi_{y}
$$

with couplings $\rho_{\mu}(x-y)$ calculable by a Fourier transformation. This lattice action is perfect by construction, i.e. its spectrum is identical with the one of the continuum theory. Hence, there should be no fermion doubling. On the other hand, the action is manifestly chirally invariant. This seems to contradict the Nielsen-Ninomiya theorem. However, the theorem is evaded because the action turns out to be nonlocal. Its couplings $\rho_{\mu}(x-y)$ do not decay exponentially at large distances. Instead for $d \geq 2$ they decay only power-like. As a consequence, in momentum space $\rho_{\mu}(p)$ is not regular (it actually has poles) and therefore the topological arguments behind the Nielsen-Ninomiya theorem do not apply. The nonlocality can be seen easily for $d=1$. Then $\gamma_{1}=1$ and the sum in eq.(53) can be performed analytically, resulting in a massless propagator that takes the form

$$
\langle\bar{\Psi}(-p) \Psi(p)\rangle=\sum_{l \in \mathbf{Z}}[i(p+2 \pi l / a)]^{-1} \Pi(p+2 \pi l / a)^{2}=\frac{a}{2 i} \cot \left(\frac{p a}{2}\right) .
$$

This implies

$$
\rho_{1}(p)=\frac{2}{a} \tan \left(\frac{p a}{2}\right)
$$

which is singular at the edge of the Brillouin zone $(p= \pm \pi / a)$. The corresponding coupling in coordinate space,

$$
\rho_{1}(x-y)=\frac{1}{a}(-1)^{(x-y) / a},
$$

does not decay at all at large distances $x-y$ and thus describes an extremely nonlocal action. For $d \geq 2$ the chirally symmetric perfect action remains nonlocal with a power-law decay of the couplings at large distances.

Although the nonlocality of the perfect action arose naturally by blocking the theory from the continuum, from a practical point of view it is very inconvenient. For example, in a numerical simulation 
it would be very demanding to include couplings to far away neighbors. It follows from the NielsenNinomiya theorem that, in order to obtain a local perfect action, one must break chiral symmetry explicitly. This can be done by modifying the above way of blocking from the continuum which was chirally covariant. If one chooses to break chiral symmetry explicitly in the blocking procedure, the resulting perfect lattice action is not manifestly chirally invariant, but it is local. This can be achieved by constructing a perfect lattice action $S[\bar{\Psi}, \Psi]$ as

$$
\begin{aligned}
\exp (-S[\bar{\Psi}, \Psi]) & =\int \mathcal{D} \bar{\psi} \mathcal{D} \psi \exp \left\{-\frac{1}{(2 \pi)^{d}} \int d^{d} p \bar{\psi}(-p)\left[i \gamma_{\mu} p_{\mu}+m\right] \psi(p)\right\} \\
& \times \exp \left\{-\frac{1}{c} \frac{1}{(2 \pi)^{d}} \int_{B} d^{d} p\left[\bar{\Psi}(-p)-\sum_{n \in \mathbf{Z}^{d}} \bar{\psi}(-p-2 \pi n / a) \Pi(p+2 \pi n / a)\right]\right. \\
& \times\left[\Psi(p)-\sum_{l \in \mathbf{Z}^{d}} \psi(p+2 \pi l / a) \Pi(p+2 \pi l / a)\right] .
\end{aligned}
$$

The coefficient $c$ is a source of explicit chiral symmetry breaking, which is injected into the theory via the renormalization group transformation that maps the continuum theory to the lattice theory. For $c \rightarrow 0$ we recover the chirally invariant but nonlocal perfect lattice action from before. In general one obtains

$$
\langle\bar{\Psi}(-p) \Psi(p)\rangle=\sum_{l \in \mathbf{Z}^{d}}\left[i \gamma_{\mu}\left(p_{\mu}+2 \pi l_{\mu} / a\right)+m\right]^{-1} \Pi(p+2 \pi l / a)^{2}+c,
$$

which corresponds to a local perfect action as long as $c \neq 0$.

Let us vary $c$ in order to optimize the locality of the perfect action. For this purpose we again consider $d=1$. Then the sum in eq.(59) can be performed analytically and the fermion propagator takes the form

$$
\langle\bar{\Psi}(-p) \Psi(p)\rangle=\frac{1}{m}-\frac{2}{m^{2} a}\left[\operatorname{coth}\left(\frac{m a}{2}\right)-i \cot \left(\frac{p a}{2}\right)\right]^{-1}+c .
$$

If we choose

$$
c=\frac{\exp (m a)-1-m a}{m^{2} a}
$$

the propagator reduces to

$$
\langle\bar{\Psi}(-p) \Psi(p)\rangle=\left(\frac{\exp (m a)-1}{m a}\right)^{2}\left[i \frac{1}{a} \sin (p a)+\frac{\exp (m a)-1}{a}+\frac{2}{a} \sin ^{2}\left(\frac{p a}{2}\right)\right]^{-1} .
$$

This corresponds to the standard Wilson fermion action except that the mass $m$ is now replaced by $(\exp (m a)-1) / a$. Hence, for the above choice of $c$, in one dimension the perfect action is ultralocal, i.e. it has only nearest-neighbor interactions. In the massless limit $m=0$ the optimal choice for locality is $c=a / 2$. When we go to more than one dimension the action remains local, but it is no longer ultralocal.

Next we derive the energy-momentum dispersion relation of perfect lattice fermions. The fermion 2-point function takes the form

$$
\begin{aligned}
\left\langle\bar{\Psi}(-\vec{p}, 0) \Psi\left(\vec{p}, x_{d}\right)\right\rangle & =\frac{1}{2 \pi} \int_{-\pi / a}^{\pi / a} d p_{d}\langle\bar{\Psi}(-p) \Psi(p)\rangle \exp \left(i p_{d} x_{d}\right) \\
& =\frac{1}{2 \pi} \int_{-\pi / a}^{\pi / a} d p_{d}\left\{\sum_{l \in \mathbf{Z}^{d}}\left[i \gamma_{\mu}\left(p_{\mu}+2 \pi l_{\mu} / a\right)+m\right]^{-1} \Pi(p+2 \pi l / a)^{2}+c\right\} \exp \left(i p_{d} x_{d}\right) \\
& =\frac{1}{2 \pi} \int_{-\infty}^{\infty} d p_{d} \sum_{l \in \mathbf{Z}^{d-1}} \frac{m}{(\vec{p}+2 \pi \vec{l} / a)^{2}+p_{d}^{2}+m^{2}}
\end{aligned}
$$




$$
\begin{aligned}
& \times \prod_{i=1}^{d-1}\left(\frac{2 \sin \left(p_{i} a / 2\right)}{p_{i} a+2 \pi l_{i}}\right)^{2}\left(\frac{2 \sin \left(p_{d} a / 2\right)}{p_{d} a}\right)^{2} \exp \left(i p_{d} x_{d}\right)+c \delta_{x_{d}, 0} \\
& =\sum_{\vec{l} \in \mathbf{Z}^{d-1}} C(\vec{p}+2 \pi \vec{l} / a) \exp \left(-E(\vec{p}+2 \pi \vec{l} / a) x_{d}\right)+c \delta_{x_{d}, 0} .
\end{aligned}
$$

The sum over $l_{d}$ has been combined with the integral of $p_{d}$ over $[-\pi / a, \pi / a]$ to an integral over the momentum space of the continuum theory. The sum over the spatial $\vec{l} \in \mathbf{Z}^{d-1}$ leads to infinitely many poles of the integrand, and hence to infinitely many states that contribute an exponential to the 2-point function. The energies of these states are given by the location of the poles, $E(\vec{p}+2 \pi \vec{l} / a)=-i p_{d}$, with

$$
E(\vec{p}+2 \pi \vec{l} / a)^{2}=-p_{d}^{2}=(\vec{p}+2 \pi \vec{l} / a)^{2}+m^{2}
$$

Hence, the energy-momentum dispersion relation of perfect lattice fermions is exactly the same as in the continuum. In particular, as a result of exact blocking from the continuum, there are no lattice artifacts. Furthermore, the form of the renormalization group blocking transformation has no effect on the physical spectrum. In particular, the explicit chiral symmetry breaking term proportional to $c$ only leads to a contact term $c \delta_{x_{d}, 0}$ in the 2-point function. Hence, it has no effect on the spectrum which is extracted from the 2-point function at large Euclidean time separations $x_{d}$. Remarkably, the spectrum of the lattice theory displays the consequences of Poincaré invariance despite the fact that the lattice action only has the discrete lattice symmetries.

Chiral symmetry is hidden in a similar way. Due to the explicit chiral symmetry breaking parameter $c$ in the renormalization group blocking transformation, even for $m=0$ the perfect lattice action is not manifestly chirally invariant. Still, all physical consequences of chiral symmetry are correctly reproduced by the perfect action. As we will see later, this is due to the by now famous Ginsparg-Wilson relation [17],

$$
\left\{D^{-1}, \gamma_{5}\right\}=a \gamma_{5}
$$

Here $D$ is the lattice Dirac operator and $D^{-1}$ is the lattice fermion propagator. Indeed, using the optimal parameter $c=a / 2$ for the perfect action one obtains

$$
\left\{D^{-1}, \gamma_{5}\right\}=\left\{\sum_{l \in \mathbf{Z}^{d}}\left[i \gamma_{\mu}\left(p_{\mu}+2 \pi l_{\mu} / a\right)\right]^{-1} \Pi(p+2 \pi l / a)^{2}+c, \gamma_{5}\right\}=2 c \gamma_{5}=a \gamma_{5}
$$

The Ginsparg-Wilson relation is the key to understanding chiral symmetry on the lattice 14. In the continuum, chiral symmetry implies $\left\{D^{-1}, \gamma_{5}\right\}=0$. If one insists on this relation also on the lattice, i.e. if one insists on manifest chiral symmetry for a lattice action, the Nielsen-Ninomiya theorem implies fermion doubling (or, even worse, a violation of locality). The Ginsparg-Wilson relation $\left\{D^{-1}, \gamma_{5}\right\}=a \gamma_{5}$ reduces to the relation $\left\{D^{-1}, \gamma_{5}\right\}=0$ in the continuum limit $a \rightarrow 0$. Still, at finite lattice spacing $a$, the right-hand side of the Ginsparg-Wilson relation implies an explicit breaking of chiral symmetry. In the case of the perfect action the explicit breaking is due to the parameter $c=a / 2$ in the renormalization group blocking transformation. This minimal explicit violation of chiral symmetry is sufficient to evade the Nielsen-Ninomiya theorem, and thus to avoid fermion doubling. Still, as we have seen explicitly for the perfect action, the physics (for example, the spectrum) remains the same as in the continuum. We will see later that the Ginsparg-Wilson relation leads to a natural definition of chiral symmetry on the lattice which reduces to the usual one in the continuum limit.

\section{Lattice QCD}

While many properties of lattice chiral symmetry can be studied in the free theory, one certainly also needs to understand it in the interacting theory. Hence, it is now time to endow the quarks with their nontrivial QCD dynamics by coupling them to the gluon field. However, before doing so, we first discuss lattice Yang-Mills theory without quarks. 


\subsection{Lattice Yang-Mills Theory}

Maintaining manifest gauge invariance is essential when gauge theories are regularized on the lattice. In the continuum, gauge transformations involve space-time derivatives of group-valued functions $\Omega(x)$. On the lattice there are no infinitesimally close points, and continuum derivatives are usually simply replaced by finite differences. However, in order to maintain gauge invariance, one must proceed more carefully. Wegner and Wilson, as well as Smit, independently introduced the concept of a parallel transporter $U_{x, \mu} \in S U\left(N_{c}\right)$ connecting neighboring lattice points $x$ and $x+\hat{\mu}$. The parallel transporter is related to an underlying continuum gauge field $A_{\mu}(x)=i g A_{\mu}^{a}(x) T^{a}$ by

$$
U_{x, \mu}=\mathcal{P} \exp \int_{0}^{a} d t A_{\mu}(x+\hat{\mu} t)
$$

where $\mathcal{P}$ denotes path-ordering. Under a non-Abelian gauge transformation the parallel transporter transforms as

$$
U_{x, \mu}^{\prime}=\Omega_{x} U_{x, \mu} \Omega_{x+\hat{\mu}}^{\dagger}
$$

Wilson has constructed the Yang-Mills part of a simple lattice QCD action by multiplying parallel transporters around an elementary plaquette. The standard Wilson action is constructed as a sum over all plaquettes

$$
S_{Y M}[U]=-a^{4} \sum_{x, \mu, \nu} \frac{1}{g^{2} a^{2}} \operatorname{Tr}\left[U_{x, \mu} U_{x+\hat{\mu}, \nu} U_{x+\hat{\nu}, \mu}^{\dagger} U_{x, \nu}^{\dagger}+U_{x, \nu} U_{x+\hat{\nu}, \mu} U_{x+\hat{\mu}, \nu}^{\dagger} U_{x, \mu}^{\dagger}\right] .
$$

It reduces to the continuum Yang-Mills action in the limit $a \rightarrow 0$.

To fully define the path integral we must also consider the measure. The lattice functional integral is obtained as an integral over all configurations of parallel transporters $U_{x, \mu}$, i.e.

$$
Z=\prod_{x, \mu} \int_{S U\left(N_{c}\right)} d U_{x, \mu} \exp \left(-S_{Y M}[U]\right)
$$

One integrates independently over all link variables using the local Haar measure $d U_{\mu, x}$ for each parallel transporter. The Haar measure is a left- and right-invariant measure, i.e.

$$
\int_{S U\left(N_{c}\right)} d U f(\Omega U)=\int_{S U\left(N_{c}\right)} d U f(U \Omega)=\int_{S U\left(N_{c}\right)} d U f(U)
$$

for any function $f(U)$ and for any $S U\left(N_{c}\right)$ matrix $\Omega$. It is convenient to normalize the measure such that

$$
\int_{S U\left(N_{c}\right)} d U=1
$$

For compact groups like $S U\left(N_{c}\right)$ the integration is over a finite domain. This makes it unnecessary to fix the gauge in lattice QCD because the functional integral is finite even without gauge fixing. This is another important advantage of the formulation using parallel transporters.

The Yang-Mills functional integral from above contains a single parameter - the bare gauge coupling $g$. When one wants to perform the continuum limit, one must search for values of $g$ for which the correlation length of the lattice theory diverges in lattice units. In the language of statistical mechanics one is looking for a second order phase transition. Due to asymptotic freedom, in lattice QCD one expects a second order phase transition at $g \rightarrow 0$. To analyze the phase structure of a gauge theory one needs to study order parameters. A simple local order parameter like $\left\langle U_{x, \mu}\right\rangle$ is not useful. This follows from Elitzur's theorem [4] which states that gauge-variant observables simply vanish. A useful order parameter in a gauge theory must be gauge invariant and, in addition, nonlocal. In a pure gluon theory a good order parameter was suggested independently by Wegner and Wilson as

$$
W_{\mathcal{C}}=\operatorname{Tr} \prod_{(x, \mu) \in \mathcal{C}} U_{x, \mu}
$$


For a rectangular curve $\mathcal{C}$ with side lengths $R$ and $T$ the Wilson loop describes the instantaneous creation and annihilation of a static quark-anti-quark pair at distance $R$ which then exists for a time $T$. The Wilson loop is related to the static quark-anti-quark potential $V(R)$ by

$$
\lim _{T \rightarrow \infty}\left\langle W_{\mathcal{C}}\right\rangle \sim \exp (-V(R) T)
$$

In QCD we expect quarks and anti-quarks to be confined to one another by a potential rising linearly at large separations $R$, i.e.

$$
\lim _{R \rightarrow \infty} V(R) \sim \sigma R
$$

where $\sigma$ is the string tension. In a confinement phase the Wilson loop hence shows an area law

$$
\lim _{R, T \rightarrow \infty}\left\langle W_{\mathcal{C}}\right\rangle \sim \exp (-\sigma R T)
$$

Confinement is indeed verified very accurately in numerical simulations of lattice Yang-Mills theories.

\subsection{Standard Wilson Action for Lattice QCD}

We still need to couple the quarks to the gluons. First we do this by gauging $S U\left(N_{c}\right)$ in the action of free Wilson fermions of eq.(45)

$$
\begin{aligned}
S_{Q C D}[\bar{\Psi}, \Psi, U] & =a^{4} \sum_{x, \mu} \frac{1}{2 a}\left(\bar{\Psi}_{x} \gamma_{\mu} U_{x, \mu} \Psi_{x+\hat{\mu}}-\bar{\Psi}_{x+\hat{\mu}} \gamma_{\mu} U_{x, \mu}^{\dagger} \Psi_{x}\right)+a^{4} \sum_{x} m \bar{\Psi}_{x} \Psi_{x} \\
& +a^{4} \sum_{x, \mu} \frac{1}{2 a}\left(2 \bar{\Psi}_{x} \Psi_{x}-\bar{\Psi}_{x} U_{x, \mu} \Psi_{x+\hat{\mu}}-\bar{\Psi}_{x+\hat{\mu}} U_{x, \mu}^{\dagger} \Psi_{x}\right) \\
& -a^{4} \sum_{x, \mu, \nu} \frac{1}{g^{2} a^{2}} \operatorname{Tr}\left[U_{x, \mu} U_{x+\hat{\mu}, \nu} U_{x+\hat{\nu}, \mu}^{\dagger} U_{x, \nu}^{\dagger}+U_{x, \nu} U_{x+\hat{\nu}, \mu} U_{x+\hat{\mu}, \nu}^{\dagger} U_{x, \mu}^{\dagger}\right] .
\end{aligned}
$$

In order to eliminate the doubler fermions we have introduced the Wilson term which breaks chiral symmetry explicitly. The lattice regularized functional integral takes the form

$$
Z=\prod_{x} \int d \bar{\Psi}_{x} d \Psi_{x} \prod_{x, \mu} \int_{S U\left(N_{c}\right)} d U_{x, \mu} \exp \left(-S_{Q C D}[\bar{\Psi}, \Psi, U]\right)
$$

It depends on two parameters - the bare gauge coupling $g$ and the bare quark mass $m$. Due to asymptotic freedom, in order to reach the continuum limit one must take $g \rightarrow 0$. When one puts $m=0$ for free Wilson fermions one reaches the chiral limit. In the interacting theory, on the other hand, this is no longer the case. In particular, since chiral symmetry is explicitly broken, the bare quark mass $m$ must be fine-tuned in order to reach a massless limit. This fine-tuning is very unnatural from a theoretical point of view. In particular, following the discussion in the introduction, if one imagines Wilson's lattice QCD as an oversimplified model for the short-distance physics at the Planck scale, one could not understand at all why there are light fermions in Nature. The fine-tuning of $m$ is also inconvenient from a practical point of view. For example, in a numerical simulation of Wilson's lattice QCD one must fine-tune $m$ to many digits accuracy in order to make the pion massless. If one does this at relatively large $g$, i.e. before one reaches the continuum limit $g \rightarrow 0$, this massless "pion" is not even a proper Goldstone boson of a spontaneously broken chiral symmetry. For Wilson fermions an exact chiral symmetry that can break spontaneously does not exist at finite lattice spacing. It emerges only in the continuum limit after a delicate fine-tuning of $m$. 


\subsection{Ginsparg-Wilson Relation and Lüscher's Lattice Chiral Symmetry}

In the discussion of the perfect free fermion action we have encountered the Ginsparg-Wilson relation eq.(65). Following Lüscher [21, we will now use this relation to construct a version of chiral symmetry that is natural for a lattice theory and reduces to the usual one in the continuum limit. For this purpose we consider a lattice fermion action

$$
S[\bar{\Psi}, \Psi, U]=a^{4} \bar{\Psi} D[U] \Psi=a^{4} \sum_{x, y} \bar{\Psi}_{x} D[U]_{x, y} \Psi_{y}
$$

which is defined in terms of the lattice Dirac operator $D[U]$. This operator should be local (i.e. it should decay exponentially at large distances $x-y$ ) but not ultralocal. The lattice Dirac operator obeys the Ginsparg-Wilson relation if the corresponding fermion propagator $D[U]^{-1}$ satisfies

$$
\left\{D[U]^{-1}, \gamma_{5}\right\}=D[U]^{-1} \gamma_{5}+\gamma_{5} D[U]^{-1}=a \gamma_{5}
$$

Alternatively, the Ginsparg-Wilson relation can be written as

$$
\gamma_{5} D[U]+D[U] \gamma_{5}=a D[U] \gamma_{5} D[U]
$$

It is nontrivial to construct lattice actions that obey the Ginsparg-Wilson relation. Until now we have seen that the perfect action for massless free fermions indeed satisfies this relation. In the next two subsections we will see that the same is true for perfect actions for massless QCD as well as for overlap fermions. For the moment we don't worry about the concrete form of $D[U]$, we just assume that it obeys eq. (81).

Let us first consider an infinitesimal chiral rotation of the form familiar from the continuum

$$
\begin{aligned}
& \Psi^{\prime}=\Psi+\delta \Psi=\left(1+i \varepsilon^{a} T^{a} \gamma_{5}\right) \Psi \\
& \bar{\Psi}^{\prime}=\bar{\Psi}+\delta \bar{\Psi}=\bar{\Psi}\left(1+i \varepsilon^{a} T^{a} \gamma_{5}\right) .
\end{aligned}
$$

Here $T^{a}$ (with $a \in\left\{1,2, \ldots, N_{f}^{2}-1\right\}$ ) are the generators of $S U\left(N_{f}\right)$ and $\varepsilon^{a}$ is a small parameter. In order to discuss flavor-singlet axial transformations with an infinitesimal parameter $\varepsilon^{0}$ we also define $T^{0}=\mathbb{1}$. If the lattice action is local and has no fermion doubling, the Nielsen-Ninomiya theorem implies that it cannot be invariant under the above chiral rotations. On the other hand, the lattice fermion measure is invariant under the full chiral symmetry $U\left(N_{f}\right)_{L} \otimes U\left(N_{f}\right)_{R}$. This is very different from massless QCD in the continuum. In the continuum the action is invariant under $U\left(N_{f}\right)_{L} \otimes U\left(N_{f}\right)_{R}$ chiral transformations, while the measure is invariant only under $S U\left(N_{f}\right)_{L} \otimes S U\left(N_{f}\right)_{R} \otimes U(1)_{L=R}$. In particular, due to the anomaly the measure of the continuum theory is not invariant under flavor-singlet axial transformations, while the measure of the lattice theory is invariant.

Next we consider Lüscher's modification of the standard chiral transformation

$$
\begin{aligned}
& \Psi^{\prime}=\Psi+\delta \Psi=\left(1+i \varepsilon^{a} T^{a} \gamma_{5}\left(1-\frac{a}{2} D[U]\right)\right) \Psi \\
& \bar{\Psi}^{\prime}=\bar{\Psi}+\delta \bar{\Psi}=\bar{\Psi}\left(1+i \varepsilon^{a} T^{a}\left(1-\frac{a}{2} D[U]\right) \gamma_{5}\right) .
\end{aligned}
$$

Through $D[U]$ Lüscher's lattice version of a chiral transformation depends on the gluon field. Still, in the continuum limit $a \rightarrow 0$ it reduces to the standard chiral symmetry of the continuum theory. It is remarkable that eq.(83) is a symmetry of any lattice action that obeys the Ginsparg-Wilson relation eq.(81). This follows from

$$
\begin{aligned}
\bar{\Psi}^{\prime} D[U] \Psi^{\prime} & =\bar{\Psi}\left(1+i \varepsilon^{a} T^{a}\left(1-\frac{a}{2} D[U]\right) \gamma_{5}\right) D[U]\left(1+i \varepsilon^{a} T^{a} \gamma_{5}\left(1-\frac{a}{2} D[U]\right)\right) \Psi \\
& =\bar{\Psi} D[U] \Psi+\bar{\Psi}\left(i \varepsilon^{a} T^{a}\left[\gamma_{5} D[U]+D[U] \gamma_{5}-a D[U] \gamma_{5} D[U]\right]\right) \Psi+O\left(\varepsilon^{2}\right) \\
& =\bar{\Psi} D[U] \Psi+O\left(\varepsilon^{2}\right) .
\end{aligned}
$$


Similarly, the variation of the lattice fermion measure takes the form

$$
\begin{aligned}
\mathcal{D} \bar{\Psi}^{\prime} \mathcal{D} \Psi^{\prime} & =\mathcal{D} \bar{\Psi}\left(1-i \varepsilon^{a} T^{a}\left(1-\frac{a}{2} D[U]\right) \gamma_{5}\right)\left(1-i \varepsilon^{a} T^{a} \gamma_{5}\left(1-\frac{a}{2} D[U]\right)\right) \mathcal{D} \Psi \\
& =\mathcal{D} \bar{\Psi} \mathcal{D} \Psi\left(1-i \varepsilon^{a} \operatorname{Tr}\left[T^{a} \gamma_{5}(2-a D[U])\right]\right)+O\left(\varepsilon^{2}\right) \\
& =\mathcal{D} \bar{\Psi} \mathcal{D} \Psi\left(1+i \varepsilon^{0} a \operatorname{Tr}\left[\gamma_{5} D[U]\right]\right)+O\left(\varepsilon^{2}\right) .
\end{aligned}
$$

Hence, while any Ginsparg-Wilson fermion action is invariant under Lüscher's lattice chiral symmetry, the lattice fermion measure is not. Exactly as in the continuum, the fermionic measure of the lattice theory changes under flavor-singlet axial transformations, while it is invariant under $S U\left(N_{f}\right)_{L} \otimes S U\left(N_{f}\right)_{R} \otimes$ $U(1)_{L=R}$. We will see later that the non-invariance of the fermionic measure under flavor-singlet axial transformations indeed gives rise to the correct axial anomaly.

\subsection{Classically Perfect Action for Lattice QCD}

Hasenfratz and Niedermayer have initiated and carried out an impressive program of explicitly constructing nonperturbative lattice actions that are perfect at least at the classical level [42. The pure gauge part of a classically perfect action is the fixed point of a renormalization group blocking transformation that maps lattice gauge fields $U$ on a fine lattice with lattice spacing a to lattice gauge fields $U^{\prime}$ on a coarser lattice with spacing $2 a$. Denoting the blocking kernel by $T_{Y M}\left[U, U^{\prime}\right]$, the classically perfect action obeys the condition

$$
S_{Y M}\left[U^{\prime}\right]=\min _{U}\left(S_{Y M}[U]+T_{Y M}\left[U, U^{\prime}\right]\right)
$$

which implicitly defines $S[U]$. This equation can be solved numerically by an iterative procedure on a multi-layer of finer and finer lattices.

The fermionic part of a classically perfect massless fermion action results as a fixed point of a renormalization group transformation which maps a fermion field $\Psi$ on the fine lattice to a fermion field $\Psi^{\prime}$ on the coarse lattice using a blocking kernel $T_{F}[U]$, i.e.

$$
\Psi^{\prime}=T_{F}[U] \Psi \Rightarrow \Psi_{x^{\prime}}^{\prime}=\sum_{x} T_{F}[U]_{x^{\prime}, x} \Psi_{x}
$$

Here $x$ is a point on the fine lattice and $x^{\prime}$ is a point on the coarse lattice. The fermionic part of the classically perfect action is quadratic in the fermion fields and can be written as

$$
S_{F}[\bar{\Psi}, \Psi, U]=a^{4} \bar{\Psi} D_{P}[U] \Psi=a^{4} \sum_{x, y} \bar{\Psi}_{x} D_{P}[U]_{x, y} \Psi_{y}
$$

The corresponding fermion propagator $D_{P}[U]^{-1}$ obeys the equation

$$
D_{P}\left[U^{\prime}\right]^{-1}=T_{F}[U] D_{P}[U]^{-1} T_{F}[U]+c \Rightarrow D_{P}\left[U^{\prime}\right]_{x^{\prime}, y^{\prime}}^{-1}=\sum_{x, y} T_{F}[U]_{x^{\prime}, x} D_{P}[U]_{x, y}^{-1} T_{F}[U]_{y, y^{\prime}}+c \delta_{x^{\prime}, y^{\prime}}
$$

Here $U$ is the gauge field on the fine lattice that minimizes the expression on the right-hand side of eq. (86) given a gauge field $U^{\prime}$ on the coarse lattice. The parameter $c$ is analogous to the one introduced in the perfect action of the free theory. For an appropriate fermionic blocking kernel $T_{F}[U]$ and a choice of $c$ that again optimizes locality one can show that the classically perfect action obeys the Ginsparg-Wilson relation.

There are a number of important properties of the classically perfect fixed point action. For example,

(i) The free gauge and fermion parts of the action reproduce the exact relativistic spectrum of the continuum. 
(ii) It is possible to define a topological charge such that the action of any gauge field configuration with topological charge $Q$ is larger than $8 \pi^{2}|Q| / g^{2}$, a relation that is known from the continuum theory.

(iii) There is an exact index theorem on the lattice that relates the fermionic zero modes of the fixed point Dirac operator and the topological charge of the lattice gauge field.

(iv) Due to the Ginsparg-Wilson relation there is an exact chiral symmetry.

For more details we refer the reader to the article by Hasenfratz [16] and references therein.

Although classically perfect actions satisfy a number of attractive properties, they are difficult to implement in practice. A highly nontrivial and crucial step is to find practical (but still accurate) parameterizations of the perfect action. Although the initial work involved spin and gauge models in two dimensions, recently practical approximations of the fixed point actions for lattice QCD have emerged. In the pure gauge theory a useful parametrization was found in [10], while the fermionic problem was tackled in [15. The resulting approximations of classically perfect actions yield physical results that are almost completely free of lattice artifacts [43, 44].

\subsection{Domain Wall and Overlap Fermions}

In the early nineties Kaplan proposed a novel method to preserve chirality on the lattice [5]. The idea was to use the fact that chiral fermions become trapped on domain walls [4. Kaplan used a Wilson-Dirac operator in five dimensions with a mass term that is a function of the fifth direction. In particular, the mass term changes sign creating a four-dimensional domain wall at the points where it vanishes. A four-dimensional chiral fermion is then trapped on the domain wall. In the meantime Narayanan and Neuberger were developing an idea of using an infinite number of regulator "flavor" fields to preserve chirality [6]. They realized that Kaplan's construction was equivalent to their idea since the fifth dimension is analogous to a flavor space. They used their interpretation and argued that the determinant of a chiral fermion in the background of a gauge field is equivalent to the overlap of two many-body fermionic ground states [8]. Initially, it seemed that the overlap was a reliable technique to regulate even chiral gauge theories on a lattice. Unfortunately, it was realized that the fermions with opposite chirality (which originate from an anti-wall) cannot be easily decoupled [45, 46.

Although it was not clear whether the domain wall and overlap approach gave a completely satisfactory formulation of lattice chiral gauge theories involving no doublers of opposite chirality, something highly nontrivial had been achieved. It was possible to construct a lattice theory with a fermion with opposite chirality and use it effectively to preserve chiral symmetry in a vector-like gauge theory. An elegant way to use a five-dimensional fermion action to represent quarks in lattice QCD was first proposed by Shamir 47] and elaborated further by Furman and Shamir in 48]. This fermion is commonly referred to as a domain wall fermion and is used extensively in lattice simulations. Its action is constructed on a five-dimensional space-time lattice with coordinates $\left(x, x_{5}\right)$, where $x$ refers to the usual four dimensions and $x_{5} \in\left\{a_{5}, 2 a_{5}, \ldots, L_{5}\right\}$ refers to the fifth direction of finite extent $L_{5}$. Since the fifth direction is physically different from the other directions we have introduced a new lattice spacing $a_{5}$ in that direction. The domain wall fermion action is given by

$$
S_{F}[\bar{\Psi}, \Psi, U]=a^{4} a_{5} \sum_{x, x_{5}, y, y_{5}} \bar{\Psi}_{x, x_{5}} D_{D W}[U]_{x, x_{5} ; y, y_{5}} \Psi_{y, y_{5}} .
$$

The domain wall Dirac operator is given by

$$
\begin{aligned}
D_{D W}[U]_{x, x_{5} ; y, y_{5}} & =\delta_{x_{5}, y_{5}} D^{\|}[U]_{x, y}+\delta_{x, y} D^{\perp}[U]_{x_{5}, y_{5}}, \\
D^{\|}[U]_{x, y} & =M \delta_{x, y}+\sum_{\mu} \frac{1}{2 a}\left(\gamma_{\mu} U_{x, \mu} \delta_{x+\hat{\mu}, y}-\gamma_{\mu} U_{x-\hat{\mu}, \mu}^{\dagger} \delta_{x-\hat{\mu}, y}\right)
\end{aligned}
$$




$$
\begin{aligned}
&- \sum_{\mu} \frac{1}{2 a}\left(2 \delta_{x, y}-U_{x, \mu} \delta_{x+\hat{\mu}, y}-U_{x-\hat{\mu}, \mu}^{\dagger} \delta_{x-\hat{\mu}, y}\right), \\
& D^{\perp}[U]_{x_{5}, y_{5}}= \begin{cases}\left(P_{R} \delta_{2 a_{5}, y_{5}}-\delta_{a_{5}, y_{5}}\right) / a_{5}-m P_{L} \delta_{L_{5}, y_{5}} & \text { for } x_{5}=1, \\
\left(P_{R} \delta_{x_{5}+a_{5}, y_{5}}+P_{L} \delta_{x_{5}-a_{5}, y_{5}}-\delta_{x_{5}, y_{5}}\right) / a_{5} & \text { for } a_{5}<s<L_{5}, \\
\left(P_{L} \delta_{L_{5}-a_{5}, y_{5}}-\delta_{L_{5}, y_{5}}\right) / a_{5}-m P_{R} \delta_{a_{5}, y_{5}} & \text { for } x_{5}=L_{5} .\end{cases}
\end{aligned}
$$

Here $P_{R}$ and $P_{L}$ are the chiral projection operators defined in eq.(18). In the above action the parameter $M$ is not the mass of the quark that is bound to the wall. By comparing with Wilson fermions one sees that the sign of the Wilson term has changed. In order to produce massless quarks one should set $0 \leq M a_{5} \leq 2$ at tree level [5] and take $L_{5} \rightarrow \infty$. There is a technical problem that needs to be taken into account. When $L_{5}$ becomes infinite there are only $N_{f}$ flavors of four-dimensional massless quarks bound to the wall, but there is an infinite number of modes at the cut-off. This may cause spurious effects at low energies. Hence, one needs to use bosonic (Pauli-Villars type) fields to cancel the contribution of these high-energy modes. For a detailed discussion of how this can be accomplished we refer the reader to [48].

There is a close connection between the domain wall approach and the overlap formula developed by Narayanan and Neuberger. Neuberger realized that it is possible to find an analytic formula for an effective Dirac operator that describes the massless chiral mode of the domain wall fermion. Using his insight on the overlap formula for vector-like gauge theories [49], he found a simple and elegant formula for the four-dimensional Dirac operator [18, which is referred to as the overlap Dirac operator and which is given by

$$
D_{O}[U]=\frac{1}{2 a}\left[1+\gamma_{5} \frac{H[U]}{\sqrt{H[U]^{2}}}\right]
$$

where $H[U]=\gamma_{5} D^{\|}[U]$ and $D^{\|}[U]$ is the operator we defined above in the context of the domain wall fermion. In order to obtain massless quarks one needs to set $0 \leq M a_{5} \leq 2$ as before. In fact, it is possible to find an analytic formula for an effective Dirac operator that represents the chiral massless modes of the domain wall fermion even for finite $L_{5}$ [49, 19, 50, 51]. This operator takes the form

$$
D_{L_{5}}[U]=\frac{1}{a}\left[1+\gamma_{5} \frac{(1+\tilde{H}[U])^{L_{5} / a_{5}}-(1-\tilde{H}[U])^{L_{5} / a_{5}}}{(1+\tilde{H}[U])^{L_{5} / a_{5}}+(1-\tilde{H}[U])^{L_{5} / a_{5}}}\right],
$$

where

$$
\tilde{H}[U]=\gamma_{5} \tilde{X}[U], \quad \tilde{X}[U]=\frac{a_{5} D^{\|}[U]}{2+a_{5} D^{\|}[U]} .
$$

In the limit of $L_{5} \rightarrow \infty$ one obtains

$$
\lim _{L_{5} \rightarrow \infty} D_{L_{5}}[U]=D_{D W O}[U]=\frac{1}{2 a}\left[1+\gamma_{5} \frac{\tilde{H}[U]}{\sqrt{\tilde{H}[U]^{2}}}\right],
$$

which reduces to the overlap Dirac operator $D_{O}[U]$ when $a_{5} \rightarrow 0$.

Although today we know that the Ginsparg-Wilson relation leads to an exact chiral symmetry on the lattice, this connection was not appreciated until recently. After Ginsparg and Wilson discovered this interesting relation they found it difficult to explicitly construct a local operator that satisfies it. The relation was soon forgotten. After the discovery of perfect and overlap fermions the relation was rediscovered by Hasenfratz. It is straightforward to check that $D_{O}[U]$ and $D_{D W O}[U]$ indeed satisfy the Ginsparg-Wilson relation. These new Dirac operators couple every pair of sites on the lattice. It is possible to show that if one wants to benefit from good chiral properties of Ginsparg-Wilson fermions, one has to give up the notion of ultralocal actions [52. However, as has been shown in [53], close to the continuum limit the couplings in the overlap Dirac operator fall off exponentially with the distance. In 
this sense these new Dirac operators are still local. Unfortunately, the closeness to the continuum limit is quite important to maintain both the chiral and locality properties of the Dirac operator. Recently, a physical picture based on the locality of zero modes of the Dirac operator was used to map out the regions in coupling constant space where $D_{O}[U]$ and $D_{D W O}[U]$ lead to a good regularization of massless quarks 54 .

There have been recent efforts to generalize the Ginsparg-Wilson relation [55] and use this as a guide to construct new classes of Dirac operators [56]. Since Dirac operators which satisfy the Ginsparg-Wilson relation exactly are computationally very demanding, there have also been efforts to find perfect Dirac operators that satisfy the Ginsparg-Wilson fermions approximately [57]. Another approach has been to expand the most general lattice Dirac operator in a basis of simple operators. The coefficients of the expansion then are determined using the Ginsparg-Wilson relation [58. This approach has been used to construct a practical operator for lattice simulations [59].

\subsection{Quenched Approximation}

The chiral limit of lattice QCD is computationally very demanding because all algorithms contain a step in which the inverse of the Dirac operator in a fixed gauge field background needs to be computed. If the Dirac operator has small eigenvalues this step becomes very time consuming. Further, in the presence of small eigenvalues the number of inversions necessary before a statistically independent configuration is generated also increases leading to inefficiencies in the algorithms.

The full partition function of lattice QCD is given by

$$
\begin{aligned}
Z & =\prod_{x} \int_{d \bar{\Psi}_{x}} d \Psi_{x} \prod_{x, \mu} \int_{S U\left(N_{c}\right)} d U_{x, \mu} \exp \left(-S_{Y M}[U]\right) \exp \left(-a^{d} \bar{\Psi} D[U] \Psi\right) \\
& =\prod_{x, \mu} \int_{S U\left(N_{c}\right)} d U_{x, \mu} \exp \left(-S_{Y M}[U]\right) \operatorname{det} D[U] .
\end{aligned}
$$

Observables take the form

$$
\langle O\rangle=\frac{1}{Z} \prod_{x, \mu} \int_{S U\left(N_{c}\right)} d U_{x, \mu} \exp \left(-S_{Y M}[U]\right) \operatorname{det} D[U] O[U],
$$

where $O[U]$ is the observable in the background gauge field $U$. For example, when the observable is a fermion bilinear, $O[U]$ is constructed from a quark propagator. The difficulties in the chiral limit arise due to the determinant factors in the above relations. In order to avoid such difficulties, the determinant factor is sometimes dropped. This is an approximation in which one ignores the effects of virtual quark loops, which is commonly referred to as the quenched approximation. It should be pointed out that the quenched approximation is not a systematically controlled one. It is possible to take into account some effects of virtual quark loops by allowing the quarks in the loop to be heavier. This is the so-called partially quenched approximation. Many calculations today use either a quenched or partially quenched approximation to compute physical quantities for light quarks.

Not surprisingly, in the chiral limit the quenched approximation introduces unphysical singularities. These singularities are again related to the small eigenvalues of the Dirac operator. However, now they enter observables through the quark propagator. Using quenched chiral perturbation theory, one can predict the form of these singularities in various observables close to the chiral limit [60, 61]. For example, the chiral condensate is expected to diverge logarithmically with a dimensionless coefficient $\delta$. Such spurious divergences are referred to as quenched chiral logarithms. Recently, several groups have evaluated $\delta$ with results varying from about 0.03 to about 0.26 . For further details we refer the reader to 62 .

The quenched approximation is particularly severe in the case of Wilson fermions close to the chiral limit. This is because, due to the absence of an exact chiral symmetry, the Wilson Dirac operator may 
contain arbitrarily small eigenvalues when the quark mass is small even if it is non-zero. In the case of staggered fermions and Ginsparg-Wilson fermions one can show that the eigenvalues of the Dirac operator cannot be smaller than the quark mass, as expected in the continuum. This problem hinders simulations with dynamical light Wilson quarks.

By adding a so-called twisted mass term to the two-flavor Wilson fermion action, it is possible to regulate the small eigenvalues of the Dirac operator [63]. This term does not change the continuum limit of the theory, since one can argue that — provided the continuum limit is taken carefully — the extra term is equivalent to a redefinition of the fermion field 64. This new approach to lattice QCD with an additional twisted mass term is becoming another practical approach to study the effects of light quarks in QCD, which has been tested in [65].

\section{Special Features of Ginsparg-Wilson Fermions}

We have already discussed in section 4.3 how the Ginsparg-Wilson relation leads to a new realization of chiral symmetry in a finite lattice theory. This lattice chiral symmetry makes domain wall and overlap fermions, which were introduced in section 4.5, special in various ways. In this section we discuss some of the features of these Ginsparg-Wilson fermions that allow us to relate lattice quantities to continuum physical quantities more directly than it was possible before.

\subsection{Anomaly and Topological Charge on the Lattice}

It is well-known that the flavor-singlet chiral symmetry is anomalous in QCD. This can be shown in the continuum in essentially two ways: (i) By taking suitable care of the ultraviolet regulator in the diagrammatic approach one can show that the divergence of the flavor-singlet axial current is equal to the topological charge density of the gluon field; (ii) in the functional integral representation of QCD the fermionic integration measure is not invariant under flavor-singlet axial transformations when the Dirac operator in the background of a gluon field configuration has a non-zero index [66, 67]. Before the discovery of the Ginsparg-Wilson relation and its consequences, in a lattice formulation the anomaly could only be understood after a complex calculation [68]. Since the fermionic measure was always invariant under chiral transformations, either the anomalous symmetry was explicitly broken by the lattice action (as in the case of Wilson or staggered fermions) or the fermion doubling would cancel the anomaly completely (as for naive fermions). The only viable approach was to calculate the appropriate diagrams in the lattice theory, which is usually quite tedious, and then to take the continuum limit.

The discovery of the exact chiral symmetry of Ginsparg-Wilson fermions allows one to derive the anomaly in a straightforward fashion as explained in 21. Here we review the essential steps of that proof. Consider the expectation value of an operator $\mathcal{O}[\bar{\Psi}, \Psi]$ in the background of a gauge field $U$ given by

$$
\langle\mathcal{O}\rangle=\prod_{x} \int d \bar{\Psi}_{x} d \Psi_{x} \mathcal{O}[\bar{\Psi}, \Psi] \exp \left(-a^{4} \sum_{x, y} \bar{\Psi}_{x} D_{x, y}[U] \Psi_{y}\right)
$$

where $D[U]_{x, y}$ is a lattice Dirac operator that obeys the Ginsparg-Wilson relation. For simplicity we have suppressed color, flavor, and Dirac indices. Using the variation of the fermionic measure eq. (85)

under Lüscher's infinitesimal flavor-singlet axial transformations as well as the invariance of the action one can show that

$$
\langle\delta \mathcal{O}\rangle=\varepsilon^{0} a \operatorname{Tr}\left[\gamma_{5} D[U]\right]\langle\mathcal{O}\rangle .
$$

As argued in [21, if $D[U]$ obeys the Ginsparg-Wilson relation it is possible to show that

$$
a(z-D[U]) \gamma_{5}(z-D[U])=z(2-a z) \gamma_{5}-(1-a z)\left[(z-D[U]) \gamma_{5}+\gamma_{5}(z-D[U])\right]
$$


where $z$ is a complex number not contained in the spectrum of $D[U]$. After multiplying both sides of this equation from the right with $(z-D[U])^{-1}$ and taking the trace one gets

$$
-a \operatorname{Tr}\left[\gamma_{5} D[U]\right]=z(2-a z) \operatorname{Tr}\left[\gamma_{5}(z-D[U])^{-1}\right]
$$

We can now divide both sides by the factor $z(2-a z)$ and integrate over a small circle centered at the origin that does not encircle any spectral value of $D[U]$ other than 0 . Since

$$
P_{0}=\oint \frac{d z}{2 \pi i}(z-D[U])^{-1}
$$

projects on the subspace of zero modes of $D[U]$, we then get

$$
a \operatorname{Tr}\left[\gamma_{5} D[U]\right]=2\left(n_{-}-n_{+}\right)=2 \operatorname{index}(D[U])
$$

where $n_{ \pm}$represent the number of zero modes of $D[U]$ which are also eigenstates of $\gamma_{5}$ with eigenvalues \pm 1 . This yields the anomalous Ward-identity

$$
\langle\delta \mathcal{O}\rangle=2 \operatorname{index}(D[U])\langle\mathcal{O}\rangle
$$

which is familiar from the continuum but which is now valid in a completely regularized finite lattice theory.

Often a lattice Dirac operator $D[U]$ is $\gamma_{5}$-Hermitean, i.e. it obeys the relation $D^{\dagger}=\gamma_{5} D \gamma_{5}$, in addition to satisfying the Ginsparg-Wilson relation. In that case one can simplify the above discussion which relates $a \operatorname{Tr}\left[\gamma_{5} D[U]\right]$ to the index of $D[U]$. This was done in [69, 70] where the lattice index theorem was first derived. One can show that the eigenstates $u_{\lambda}$ of $a D[U]$ are such that the eigenvalues $\lambda$ fall on a circle given by $\lambda=1-\mathrm{e}^{i \alpha}$. There are three types of eigenstates: (i) Those with $\lambda=0$ such that $\gamma_{5} u_{\lambda}= \pm u_{\lambda}$. Let $n_{ \pm}$represent the number of such eigenstates with eigenvalue \pm 1 ; (ii) those with $\lambda=2$ such that $\gamma_{5} u_{\lambda}= \pm u_{\lambda}$. Let $n_{ \pm}^{\prime}$ represent the number of such eigenstates with eigenvalue \pm 1 ; (iii) those with complex (non-real) $\lambda$, in which case $\gamma_{5} u_{\lambda}=u_{\lambda^{*}}$. Since $\operatorname{Tr}\left(\gamma_{5}\right)=0$, we must have $n_{-}-n_{+}=n_{+}^{\prime}-n_{-}^{\prime}$. Using these relations it is easy to derive eq.(103).

The above calculation shows that there is indeed an anomaly in a theory with Ginsparg-Wilson fermions. However, it is also important to show that the anomaly reproduces the well-known results in the continuum limit. This has been shown perturbatively for a class of overlap Dirac operators in 71] and nonperturbatively for the overlap Dirac operator of eq. (92) in [72, 73, 74, 75. More recently, the nonperturbative arguments were extended to the overlap Dirac operator that is constructed using a perfect fermion action [76. Thus, from a variety of studies we can conclude with confidence that the above calculation does indeed reproduce the correct anomaly in the continuum limit.

In the continuum, the index of the Dirac operator is related to the topological charge of the background gauge field configuration. On the lattice there is no unique way to define the topological charge. A naive discretization of the topological charge does not even yield an integer on the lattice. A geometric construction of the topological charge is possible which then leads to integer values [77]. The index of a Ginsparg-Wilson Dirac operator is yet another definition of the topological charge. In this case we define the topological charge as

$$
Q=\frac{1}{2} a \operatorname{Tr}\left[\gamma_{5} D[U]\right]
$$

This definition is more meaningful since it is the same charge that enters the anomalous Ward identity in eq.(104). As we will see in the next section, the Witten-Veneziano mass formula can be derived on the lattice using this definition of the topological charge. Further discussions of the anomaly and the topological charge can be found in a recent review [70] and the references therein. 


\subsection{Witten-Veneziano Mass Formula on the Lattice}

The mass of the $\eta^{\prime}$-meson is closely connected to the anomalous flavor-singlet chiral symmetry of QCD. In the absence of the anomaly one can argue that the flavor-singlet chiral symmetry would be spontaneously broken leading to a massless Goldstone boson for massless quarks. The absence of a low-mass flavor-singlet pseudo-scalar particle in Nature with the correct flavor quantum numbers is usually attributed to the anomaly. To compute this mass from first principles is one of the outstanding challenges in QCD. Due to the nonperturbative nature of the physics involved, the only viable approach to this problem from first principles is using lattice QCD.

Interestingly, the physics of the $\eta^{\prime}$-meson mass can be understood more easily in certain limits of QCD. For example, in the limit $N_{c} \rightarrow \infty$, with $g^{2} N_{c}$ and $N_{f}$ held fixed [78], or by assuming that the anomalous flavor-singlet axial Ward identities retain their validity order by order in an expansion in $u \equiv N_{f} / N_{c}$ around $u=0$ [79]. In both cases one can derive a leading-order Witten-Veneziano relation for the mass of the $\eta^{\prime}$-meson which is given by

$$
m_{\eta^{\prime}}^{2}=\frac{2 N_{f}}{F_{\pi}^{2}} \int d^{4} x\langle q(x) q(0)\rangle_{Y M},
$$

where $F_{\pi}$ is the pion decay constant and $q(x)$ is the topological charge density

$$
q(x)=-\frac{1}{32 \pi^{2}} \epsilon_{\mu \nu \rho \sigma} \operatorname{Tr}\left[F_{\mu \nu}(x) F_{\rho \sigma}(x)\right] .
$$

Here the trace is over color indices. The subscript $Y M$ in eq.(106) indicates that the $q q$-correlation function is to be computed in the pure Yang-Mills theory, i.e. in the absence of quarks.

Although the Witten-Veneziano relation is valid only in certain limits of QCD, one can use it to estimate $m_{\eta^{\prime}}$ in a lattice QCD calculation. Unfortunately, eq.(106) is rather formal and cannot be translated to lattice QCD without addressing a number of subtleties [80 ${ }^{1}$. Two main problems need to be solved in order to make eq.(106) rigorous and of practical use. One has to: (i) find a properly normalized lattice definition of the topological charge density $q(x)$; (ii) subtract from $q(x) q(0)$ appropriate contact terms, so as to define it properly in the continuum limit. As we have discussed earlier, different lattice definitions of $q(x)$ are possible. Furthermore, the second problem can be quite subtle since the subtraction may involve contact terms of the form $c \delta(x)$ which contribute a finite constant to the $\eta^{\prime}$-meson mass. Some of these subtleties were first pointed out in 82. The reader is also referred to a recent discussion in [83].

One of the special features of Ginsparg-Wilson fermions is that we can use eq.(105) to define the topological charge density $q_{x}$ (which obeys $a^{4} \sum_{x} q_{x}=Q$ ) as

$$
q_{x}=\frac{1}{2 a^{3}} \operatorname{Tr}\left[\gamma_{5} D[U]_{x, x}\right] .
$$

In this expression the trace is only over the color and Dirac indices, but not over the space-time index $x$. As explained in [81], this definition allows one to derive

$$
m_{\eta^{\prime}}^{2}=\frac{2 N_{f}}{F_{\pi}^{2}} a^{4} \sum_{x}\left\langle q_{0} q_{x}\right\rangle_{Y M},
$$

in the appropriate limit in lattice QCD. Thus one obtains a relation for the $\eta^{\prime}$-meson mass exactly as in the continuum. Thanks to the chiral symmetry properties of Ginsparg-Wilson fermions, no additional subtractions are necessary.

\footnotetext{
${ }^{1}$ For a recent review we refer the reader to [81] and the references therein. Here we review only some basic points.
} 


\subsection{Renormalization of Operators}

Computing the matrix elements of fermionic currents in QCD is necessary for determining a variety of physical quantities such as hadronic decay constants, electromagnetic and weak form factors, quark masses, etc. A knowledge of the lattice renormalization constants of these operators is necessary to relate the matrix elements computed using lattice simulations to the corresponding ones defined in continuum renormalization schemes, like the $\overline{M S}$ scheme, used in experimental data analysis. Recently, several groups have contributed to this subject. We refer the reader to [84 and references therein for a recent review of renormalization in lattice field theory. The existence of exact symmetries simplifies the computations of these renormalization constants enormously. Hasenfratz has discussed operator renormalization in the context of perfect actions [85. In this section, using a few examples, we illustrate how one can obtain constraints among various renormalization constants. A more general method to renormalize massless Ginsparg-Wilson fermions in lattice gauge theories has been worked out in 866. For an extensive discussion of perturbative renormalization, especially in the context of Ginsparg-Wilson fermions we refer the reader to the recent review [87] and references therein.

Consider the renormalization constants for local bilinear quark operators of the form

$$
\mathcal{O}_{\alpha}(x)=\bar{\psi}(x) \Gamma_{\alpha} \psi(x)
$$

where $\Gamma_{\alpha}$ denotes generic Dirac matrices, i.e. $1, \gamma_{5}, \gamma_{\mu}, \gamma_{\mu} \gamma_{5}, \sigma_{\mu \nu}$. Specific bilinear operators, denoted according to their Lorentz group transformations, are

$$
S(x)=\bar{\psi}(x) \psi(x), P(x)=\bar{\psi}(x) \gamma_{5} \psi(x), V_{\mu}^{a}(x)=\bar{\psi}(x) \gamma_{\mu} T^{a} \psi(x), A_{\mu}^{a}(x)=\bar{\psi}(x) \gamma_{\mu} \gamma_{5} T^{a} \psi(x) .
$$

The lattice renormalization constants can be obtained by the equation

$$
\Gamma_{\mathcal{O}}^{\overline{M S}}(p, \mu)=Z_{\mathcal{O}}(a \mu) Z_{\psi}(a \mu) \Gamma_{\mathcal{O}}^{L}(p, a)
$$

where $\Gamma_{\mathcal{O}}^{L}(p, a)$ and $\Gamma_{\mathcal{O}}^{\overline{M S}}(p, \mu)$ are the two-quark one-particle irreducible vertex functions with an insertion of $\mathcal{O}$ calculated respectively on the lattice and in the continuum using the $\overline{M S}$ renormalization scheme, and $Z_{\psi}(a \mu)$ is the wave function renormalization constant. In the continuum, chiral symmetry imposes constraints among the various renormalization constants. For example, it is well-known that $Z_{S}=Z_{P}$ and $Z_{V}=Z_{A}$. Such relations reduce the amount of work in computing the renormalization constants. On the lattice one often has to deal with mixing of operators with lower-dimensional operators leading to additional subtractions that may diverge with an inverse power of the lattice spacing. Fortunately, with Ginsparg-Wilson fermions the renormalization of operators is also quite analogous to the continuum. For example, one can argue that the relations $Z_{S}=Z_{P}$ and $Z_{V}=Z_{A}$ remain valid [88].

In order to see the usefulness of the Ginsparg-Wilson relation, let us define a new set of fermion bilinear operators

$$
\mathcal{O}_{\alpha}^{\prime}=\bar{\Psi} \Gamma_{\alpha}\left(1-\frac{a}{2} D[U]\right) \Psi
$$

where $D[U]$ is the Ginsparg-Wilson Dirac operator appearing in the fermionic action of the theory. The addition of the term depending on $D[U]$ is harmless since its contributions vanish in the continuum limit. This leads to the fact that the renormalization constants $Z_{\mathcal{O}^{\prime}}$ for the new operators are the same as $Z_{\mathcal{O}}$. In fact, these new "primed" operators are more natural in a Ginsparg-Wilson theory at finite lattice spacings. As pointed out in [89] and [90, for finite lattice spacing the spontaneous breaking of non-singlet chiral symmetry is related to the vacuum expectation value of the operator $S^{\prime}$, i.e. it occurs if $\left\langle S^{\prime}\right\rangle \neq 0$. Further, by using the infinitesimal flavor-singlet chiral transformations given in eq.([83) one can show that

$$
\delta S^{\prime}(x)=2 \varepsilon^{0} P^{\prime}(x), \delta P^{\prime}(x)=2 \varepsilon^{0} S^{\prime}(x) .
$$


Under the non-singlet chiral transformations the vector and axial currents $V_{\mu}^{\prime a}$ and $A_{\mu}^{\prime a}$ transform as

$$
\delta V_{\mu}^{\prime a}=i f^{a b c} \varepsilon^{b} A_{\mu}^{\prime c}, \delta A_{\mu}^{\prime a}=i f^{a b c} \varepsilon^{b} V_{\mu}^{\prime c} .
$$

Using the corresponding Ward identities one can show that

$$
Z_{S^{\prime}}=Z_{P^{\prime}}, Z_{V^{\prime}}=Z_{A^{\prime}}
$$

Thus, we find that $Z_{S}=Z_{P}$ and $Z_{V}=Z_{A}$.

There is also a simplification in determining the renormalization of the quark mass using GinspargWilson fermions when the bare fermion mass is introduced in the action by writing the massive Dirac operator as

$$
D_{m}[U]=D[U]+m\left(1-\frac{a}{2} D[U]\right) .
$$

The renormalization of the fermion mass is then related to that of the operator $S^{\prime}$ denoted by $Z_{S}$. In particular, it is easy to show that

$$
Z_{m}=Z_{S}^{-1}
$$

The renormalized quark mass is then obtained as $m_{\text {ren }}=Z_{m} m$.

Using these ideas, one-loop perturbative computations of these renormalization constants were performed with overlap fermions in [88, while a nonperturbative approach has been used in 91 to determine the quark condensate and quark masses. Nonperturbative renormalization has also been studied extensively with domain wall fermions, the details of which can be found in 92 .

\subsection{Lattice Simulations with Ginsparg-Wilson Fermions}

Over the past few years, numerical simulations with overlap fermions, domain wall fermions, and approximately perfect fermions have begun to appear. Computationally, these fermions are about fifty to a hundred times more expensive than conventional fermions. Thus, the most reliable results from lattice simulations using these new fermions are still obtained in the quenched approximation where the fermion determinant is ignored. In this section we review some of the recent work.

Simulations with domain wall fermions are in much better shape thanks to both a larger number of researchers working on this subject and faster computers being used in the computations. A detailed study of the chiral properties of domain wall quarks can be found in [93 and 94. Recently, an extensive calculation of quenched QCD with light quarks using overlap fermions was performed in 95. Chiral properties of overlap fermions were studied earlier in [96, 97]. The first results using approximately classically perfect fermions, which obey the Ginsparg-Wilson relation to a good accuracy, have also been published in 98 .

Calculations using domain wall fermions typically use lattices of size $24^{3} \times 40$ to $32^{3} \times 60$ at a lattice cut-off which varies from $1 / a=2 \mathrm{GeV}$ to $3 \mathrm{GeV}$. On the other hand, simulations with overlap fermions are currently done on lattices of sizes from $12^{3} \times 24,16^{3} \times 32$ to $20^{4}$ at lattice cut-offs of $1 / a=1.33$ $\mathrm{GeV}$ to $2 \mathrm{GeV}$. Results using approximately perfect fermions have been obtained on $8^{3} \times 24,12^{3} \times 24$, and $16^{3} \times 32$ lattices at lattice cut-offs $1 / a=1.3 \mathrm{GeV}, 2 \mathrm{GeV}$, and $2.5 \mathrm{GeV}$.

A variety of quantities can be calculated in lattice simulations. This includes the hadron spectrum, the topological susceptibility, the quark condensate, the pion decay constant, the average of the up and down quark masses $\bar{m}=\left(m_{u}+m_{d}\right) / 2$, the strange quark mass $m_{s}$, weak matrix elements like the kaon $B_{K}$ parameter, properties of the nucleon, such as its axial charge $g_{A}$ and the parameters that arise in quenched and unquenched chiral perturbation theory. A comparison of the results obtained in different studies indicates that there are systematic errors in many of these quantities that are presently of the order of $10-20 \%$. For example, the topological susceptibility defined as

$$
\chi_{t}=\frac{\left\langle Q^{2}\right\rangle}{V}
$$


is found to vary from about $(0.176 \mathrm{GeV})^{4}$ in 95$],(0.196 \mathrm{GeV})^{4}$ in [99, to $(0.213 \mathrm{GeV})^{4}$ in 96 . Finite temperature effects were recently studied in [100] and it was found that $\chi_{t}$ varies from about $(0.191 \mathrm{GeV})^{4}$ at $T=0.88 T_{c}$ to $(0.100 \mathrm{GeV})^{4}$ at $T=1.31 T_{c}$ where $T_{c}$ is the deconfinement temperature. Currently, several groups are performing a careful analysis of the systematic errors due to finite volume effects, finite lattice spacing errors, as well as the effects of dynamical quark loops.

Another important parameter in QCD is the chiral condensate. However, unlike $\chi_{t}$ this quantity needs a proper definition in a renormalization scheme [101. For Ginsparg-Wilson fermions a nonperturbative renormalization group invariant definition of the condensate was introduced in [91. Using this definition the condensate was found to be $(0.243(10) \mathrm{GeV})^{3}$ in 102 using overlap fermions and $(0.235(11) \mathrm{GeV})^{3}$ in 103 using the perfect action approach. When these are converted to the $\overline{\mathrm{MS}}$ scheme at a scale of $2 \mathrm{GeV}$ one obtains $(0.266(15) \mathrm{GeV})^{3}$, which is consistent with $(0.250(3) \mathrm{GeV})^{3}$ obtained in [95. These values are in agreement with the value $(0.256(8) \mathrm{GeV})^{3}$ obtained in 93] using domain wall fermions. The errors in the chiral condensate seem to be at the $4-8 \%$ level.

One of the most impressive achievements in lattice QCD is the determination of the hadron spectrum. It is indeed exciting that one can compute the masses of a variety of hadrons from first principles and compare them with experiments. In the quenched approximation this spectrum shows about $10 \%$ deviations from the experimental results [104]. These deviations appear to reduce when one introduces dynamical quarks [105]. Since most calculations are not close to the continuum limit, to some extent the deviations depend on the quantity used to determine the lattice spacing. Recently, results using chirally improved fermions in the quenched limit have begun to emerge. Results using fixed point fermions suggest that deviations from the experimental results are strongly correlated with their experimentally observed widths [98. In other words, unstable particles are more severely affected by quenching than stable ones. Using domain wall fermions, the quenched spectrum has been investigated in [106].

Several groups have recently obtained results on kaon physics using overlap and domain wall fermions. The main goal in these projects is to extract the kaon $B_{K}$ parameter, which plays a central role in understanding $C P$ violation in the kaon system. The value of $B_{K}$ depends on the renormalization scale and scheme. When evaluated at $\mu=2 \mathrm{GeV}$ in the $\overline{M S}$ scheme the value appears to be around 0.57 with an error of about $5-10 \%$. We refer the reader to the work in [107, 108, 109, 110, for further details. Quark masses have also been studied in [95, 111] using overlap fermions and in [109, 112] using domain wall fermions. The current estimates are $\bar{m}=3.5-4 \mathrm{MeV}$ and $m_{s}=99-133 \mathrm{MeV}$.

Recently, domain wall quarks have been used to compute the nucleon axial charge $g_{A}$. One such calculation finds $g_{A}=1.21(5)$ in the chiral limit. This should be compared with the experimental result $g_{A}=1.267(3)$. We refer the reader to [113] for further details and for a comparison with earlier work. There are also interesting results on the excited states of the nucleon from different types of chiral fermion actions [114, 115, 116. Finally, we like to mention that efforts to compute the parton distribution functions from lattice QCD are well-developed (see [117] for a recent review). Recently, a method to measure even generalized parton distribution functions has been proposed [118] and has been applied using domain wall fermions [119]. Such calculations have also been performed using Wilson fermions [120, 121, 122].

There are many other studies we have not touched on in this article. All these studies indicate that, with sufficient computing power, at least in the quenched approximation, computations using GinspargWilson fermions are feasible. Dynamical fermion calculations are also being envisioned. However, in the absence of an algorithmic breakthrough for dynamical fermions, it is likely that most computations will be limited to quark masses that are still too large for chiral perturbation theory to work reliably. This may keep us from studying the full dynamics of chiral symmetry from first principles for some time. 


\section{Low-Energy Effective Theories}

Due to spontaneous chiral symmetry breaking, at low energies the QCD dynamics is dominated by Goldstone bosons 123 - the pions for $N_{f}=2$. It is possible to use a low-energy effective description that only involves the Goldstone boson fields [124, 125, 126. Chiral perturbation theory provides a systematic low-energy expansion that predicts the pion dynamics based on symmetry principles and a few low-energy parameters (like the pion decay constant $F_{\pi}$ and the chiral order parameter $\langle\bar{\psi} \psi\rangle$ ) whose values can be determined either from experiments or from lattice QCD calculations [127. The low-energy interactions of pions and nucleons can be understood in baryon chiral perturbation theory [128, 129, 130, 131, 132. Effective theories that are relevant to more than one nucleon can be useful even in the absence of pions [133, 134]. For a recent review of this subject we refer the reader to [135].

Usually, low-energy effective theories are treated perturbatively directly in the continuum. In order to investigate nonperturbative effects that may arise within the effective theory, it is interesting to also regularize it on the lattice [136, 137, 138. There are efforts underway to understand nuclear matter on the lattice 139, 140, 141, starting from some effective theory. Interestingly, the nonlinear realization of chiral symmetry on the lattice does not lead to the same subtleties (like the fermion doubling problem) that one faces within the microscopic QCD theory [142].

\subsection{Effective Theory for Goldstone Bosons}

The Goldstone bosons are the lightest particles in QCD. Therefore they dominate the dynamics of the strong interactions at low energies. It is possible to use a low-energy effective description that only involves the Goldstone boson fields. This is not only true for QCD but also for any other system with a continuous global symmetry $G$ which is spontaneously broken to a subgroup $H$. The Goldstone bosons are described by fields in the coset space $G / H$ in which points are identified if they are connected by symmetry transformations of the unbroken subgroup $H$. In QCD we have $G / H=S U\left(N_{f}\right)$. Hence the Goldstone boson fields are represented by special unitary matrices $U(x) \in S U\left(N_{f}\right)$. Under global chiral rotations they transform as

$$
U(x)^{\prime}=L U(x) R^{\dagger}
$$

Goldstone bosons interact weakly at low energies. Their effective Lagrangian is constructed as a derivative expansion. The leading term of the pion effective action takes the form

$$
S[U]=\int d^{4} x\left[\frac{F_{\pi}^{2}}{4} \operatorname{Tr}\left(\partial_{\mu} U^{\dagger} \partial_{\mu} U\right)+\frac{\langle\bar{\psi} \psi\rangle}{2 N_{f}} \operatorname{Tr}\left(\mathcal{M} U^{\dagger}+U \mathcal{M}^{\dagger}\right)\right] .
$$

The first term on the right-hand side is chirally invariant. Its prefactor is the pion decay constant $F_{\pi}$ which determines the strength of the interaction between the Goldstone bosons. The second term is the chiral symmetry breaking mass term which contains the quark mass matrix. Under chiral transformations this term transforms as

$$
\operatorname{Tr}\left(\mathcal{M} U^{\prime \dagger}+U^{\prime} \mathcal{M}^{\dagger}\right)=\operatorname{Tr}\left(\mathcal{M} R U^{\dagger} L^{\dagger}+L U R^{\dagger} \mathcal{M}^{\dagger}\right)
$$

If all quark masses are equal, i.e. if $\mathcal{M}=m \mathbb{1}$, the Lagrangian is invariant against $S U\left(N_{f}\right)_{F}$ flavor rotations for which $R=L$. For a general diagonal mass matrix the flavor symmetry is reduced to $\prod_{f=1}^{N_{f}} U(1)_{f}$.

The constants $F_{\pi}$ and $\langle\bar{\psi} \psi\rangle$ determine the low-energy dynamics at leading order and enter the effective theory as a priori unknown parameters. These parameters can be determined from experiments or through nonperturbative lattice QCD calculations. Up to these two low-energy constants the Goldstone boson dynamics is completely determined by chiral symmetry. At higher energies additional terms arise in the effective theory. Again, they are restricted by chiral symmetry and they contain new low-energy 
parameters - the Gasser-Leutwyler coefficients. Chiral perturbation theory (with mass-degenerate quarks) is a systematic low-energy expansion around the classical vacuum configuration $U(x)=\mathbb{1}$. One writes

$$
U(x)=\exp \left(2 i \pi^{a}(x) T^{a} / F_{\pi}\right), a \in\left\{1,2, \ldots, N_{f}^{2}-1\right\},
$$

where $T^{a}$ are the generators of $S U\left(N_{f}\right)$, and one then expands in powers of $\pi^{a}(x)$. In this way one can derive, for example, the Gellmann-Oakes-Renner relation

$$
M_{\pi}^{2}=\frac{2 m\langle\bar{\psi} \psi\rangle}{N_{f} F_{\pi}^{2}} .
$$

\subsection{Effective Theory for Nucleons and Pions}

Chiral perturbation theory can be extended to sectors with non-zero baryon number. Nucleons enter the low-energy effective theory in the form of a Dirac spinor field $\psi(x)$ and $\bar{\psi}(x)$ that transforms as an $S U(2)_{I}$ isospin doublet. Global chiral rotations $L \otimes R \in S U(2)_{L} \otimes S U(2)_{R}$ can be realized nonlinearly on this field using the transformations

$$
\psi(x)^{\prime}=V(x) \psi(x), \bar{\psi}(x)^{\prime}=\bar{\psi}(x) V(x)^{\dagger} .
$$

The field $V(x)$ depends on $L$ and $R$ as well as on the field $U(x)$ and can be written as

$$
V(x)=R\left(R^{\dagger} L U(x)\right)^{1 / 2}\left(U(x)^{1 / 2}\right)^{\dagger}=L\left(L^{\dagger} R U(x)^{\dagger}\right)^{1 / 2} U(x)^{1 / 2} .
$$

For transformations in the unbroken isospin vector subgroup $S U(2)_{I}=S U(2)_{L=R}$ the field $V(x)$ reduces to the global flavor transformation $V(x)=L=R$. The local transformation $V(x)$ is a nonlinear representation of chiral symmetry which has the form of a local $S U(2)$ transformation, despite the fact that it represents just a global $S U(2)_{L} \otimes S U(2)_{R}$ symmetry.

In order to construct a chirally invariant (i.e. $S U(2)$ "gauge" invariant) action one needs an $S U(2)$ flavor "gauge" field. For this purpose one constructs a field $u(x) \in S U(2)$ from the pion field $U(x)$ as

$$
u(x)=U(x)^{1 / 2}
$$

The matrix $u(x)$ is located in the middle of the shortest geodesic connecting $U(x)$ with the unit-matrix $\mathbb{1}$ in the group manifold of $S U(2)$. Under chiral rotations the field $u(x)$ transforms as

$$
u(x)^{\prime}=L u(x) V(x)^{\dagger}=V(x) u(x) R^{\dagger} .
$$

The anti-Hermitean composite field

$$
v_{\mu}(x)=\frac{1}{2}\left[u(x)^{\dagger} \partial_{\mu} u(x)+u(x) \partial_{\mu} u(x)^{\dagger}\right]
$$

transforms as a "gauge" field

$$
v_{\mu}(x)^{\prime}=\frac{1}{2}\left[V(x) u(x)^{\dagger} L^{\dagger} \partial_{\mu}\left(L u(x) V(x)^{\dagger}\right)+V(x) u(x) R^{\dagger} \partial_{\mu}\left(R u(x)^{\dagger} V(x)^{\dagger}\right)\right]=V(x)\left(v_{\mu}(x)+\partial_{\mu}\right) V(x)^{\dagger} .
$$

A Hermitean composite field is given by

$$
a_{\mu}(x)=\frac{i}{2}\left[u(x)^{\dagger} \partial_{\mu} u(x)-u(x) \partial_{\mu} u(x)^{\dagger}\right]
$$

which transforms as

$$
a_{\mu}(x)^{\prime}=\frac{1}{2}\left[V(x) u(x)^{\dagger} L^{\dagger} \partial_{\mu}\left(L u(x) V(x)^{\dagger}\right)-V(x) u(x) R^{\dagger} \partial_{\mu}\left(R u(x)^{\dagger} V(x)^{\dagger}\right)\right]=V(x) a_{\mu}(x) V(x)^{\dagger} .
$$


The leading terms in the Euclidean action of a low-energy effective theory for nucleons and pions take the form

$$
\begin{aligned}
S[\bar{\psi}, \psi, U] & =\int d^{4} x\left\{M \bar{\psi} \psi+\bar{\psi} \gamma_{\mu}\left(\partial_{\mu}+v_{\mu}\right) \psi+i g_{A} \bar{\psi} \gamma_{\mu} \gamma_{5} a_{\mu} \psi\right. \\
& \left.+\frac{F_{\pi}^{2}}{4} \operatorname{Tr}\left[\partial_{\mu} U^{\dagger} \partial_{\mu} U\right]-\frac{\langle\bar{\psi} \psi\rangle}{4} \operatorname{Tr}\left[\mathcal{M} U^{\dagger}+\mathcal{M}^{\dagger} U\right]\right\}
\end{aligned}
$$

Here $M$ is the nucleon mass generated by spontaneous chiral symmetry breaking and $g_{A}$ is the coupling to the isovector axial current. It is remarkable that - thanks to the nonlinear realization of chiral symmetry - the fermion mass term is chirally invariant. This makes sense because the mass $M$ arises from the spontaneous breakdown of chiral symmetry even in the chiral limit. It is remarkable that fermions with a nonlinearly realized chiral symmetry do not contribute to anomalies [128, 143]. In the low-energy effective theory anomalies enter through the Wess-Zumino-Witten term [144, 145].

\subsection{Effective Theory for Constituent Quarks}

The chiral quark model of Georgi and Manohar [146] is formulated in terms of gluons, pions, and constituent quarks $\psi(x)$ and $\bar{\psi}(x)$ which transform in the fundamental representations of $S U\left(N_{c}\right)$ and $S U\left(N_{f}\right)$. In particular, under the nonlinearly realized $S U\left(N_{f}\right)_{L} \otimes S U\left(N_{f}\right)_{R}$ chiral symmetry the constituent quark field transforms as

$$
\psi(x)^{\prime}=V(x) \psi(x), \bar{\psi}(x)^{\prime}=\bar{\psi} V(x)^{\dagger}
$$

while under an $S U\left(N_{c}\right)$ color gauge transformation

$$
\psi(x)^{\prime}=\Omega(x) \psi(x), \bar{\psi}(x)^{\prime}=\bar{\psi} \Omega(x)^{\dagger} .
$$

The Euclidean action of the chiral quark model is given by

$$
\begin{aligned}
S[\bar{\psi}, \psi, U, A] & =\int d^{4} x\left\{M \bar{\psi} \psi+\bar{\psi} \gamma_{\mu}\left(\partial_{\mu}+v_{\mu}+A_{\mu}\right) \psi+i g_{A} \bar{\psi} \gamma_{\mu} \gamma_{5} a_{\mu} \psi\right. \\
& \left.+\frac{F_{\pi}^{2}}{4} \operatorname{Tr}\left[\partial_{\mu} U^{\dagger} \partial_{\mu} U\right]-\frac{1}{4}\langle\bar{\psi} \psi\rangle \operatorname{Tr}\left[\mathcal{M} U^{\dagger}+\mathcal{M}^{\dagger} U\right]-\frac{1}{2 g^{2}} \operatorname{Tr}\left[F_{\mu \nu} F_{\mu \nu}\right]\right\}
\end{aligned}
$$

In this case, $M$ is the constituent quark mass which is generated by spontaneous chiral symmetry breaking. It should be noted that in the effective theory the strong gauge coupling $g$ is weaker than in QCD itself. However, due to the coupling to the gluon field the constituent quarks are still confined.

The chiral quark model is based on the assumption that the energy scale for chiral symmetry breaking is larger than the one for confinement. An effective description in terms of constituent quarks which receive their mass from chiral symmetry breaking before they get confined by residual low-energy gluons should then make sense. In fact, the phenomenological success of the nonrelativistic quark model may suggest that this picture is indeed correct. In their work, Georgi and Manohar provided a framework that puts the nonrelativistic quark model on a solid field theoretical basis.

A potential problem of the chiral quark model is related to the confinement scale. The value of $g$ may be so small that there are unacceptably low-lying glueball states. For the same reason, there might be a low-temperature deconfinement phase transition in the gluon sector significantly below the finite temperature chiral phase transition. These potential problems are impossible to address quantitatively in the continuum formulation of the chiral quark model because they involve the nonperturbative dynamics of confinement. In order to be able to address these issues, it is useful to formulate the constituent quark model on the lattice. 


\subsection{Lattice Formulation of Nonlinearly Realized Chiral Symmetry}

Let us construct theories with a nonlinearly realized chiral symmetry on the lattice. The Goldstone boson field $U_{x} \in S U\left(N_{f}\right)$ naturally lives on the sites $x$ of a four-dimensional hypercubic lattice and it transforms as

$$
U_{x}^{\prime}=L U_{x} R^{\dagger}
$$

under global chiral rotations. As in the continuum, the field $u_{x} \in S U\left(N_{f}\right)$ is constructed as $u_{x}=U_{x}^{1 / 2}$ which transforms as

$$
u_{x}^{\prime}=L u_{x} V_{x}^{\dagger}=V_{x} u_{x} R^{\dagger}
$$

We now proceed to the construction of the lattice analog $V_{x, \mu} \in S U\left(N_{f}\right)$ of the continuum flavor "gauge" field $v_{\mu}(x)$ which is a flavor parallel transporter along a lattice link in the group $S U\left(N_{f}\right)$. In analogy to the continuum expression eq.(129) for $v_{\mu}(x)$ we construct

$$
\tilde{V}_{x, \mu}=\frac{1}{2 a}\left[u_{x}^{\dagger} u_{x+\hat{\mu}}+u_{x} u_{x+\hat{\mu}}^{\dagger}\right]
$$

In the continuum limit $\tilde{V}_{x, \mu}=\exp \left[a v_{\mu}(x+\hat{\mu} / 2)\right]$. However, at finite lattice spacing $\tilde{V}_{x, \mu}$ is in general not an element of $S U\left(N_{f}\right)$, just a complex $N_{f} \times N_{f}$ matrix in the group $G L\left(N_{f}\right)$. One can project a group-valued parallel transporter $V_{x, \mu} \in S U\left(N_{f}\right)$ out of $\tilde{V}_{x, \mu}$ by performing a $G L\left(N_{f}\right) / S U\left(N_{f}\right)$ coset decomposition [142]. By construction, under the nonlinearly realized chiral symmetry $V_{x, \mu}$ then transforms as a parallel transporter, i.e.

$$
V_{x, \mu}^{\prime}=V_{x} V_{x, \mu} V_{x+\hat{\mu}}^{\dagger}
$$

Next we construct a lattice version of the continuum field $a_{\mu}(x)$ defined in (131). For this purpose, we first construct

$$
\tilde{A}_{x, \mu}=\frac{i}{2 a}\left[u_{x}^{\dagger} u_{x+\hat{\mu}}-u_{x} u_{x+\hat{\mu}}^{\dagger}\right]
$$

While in the continuum $a_{\mu}(x)^{\prime}=V(x) a_{\mu}(x) V(x)^{\dagger}$, the lattice field $\tilde{A}_{x, \mu}$ transforms as

$$
\tilde{A}_{x, \mu}^{\prime}=V_{x} \tilde{A}_{x, \mu} V_{x+\hat{\mu}}^{\dagger}
$$

Also, in contrast to the continuum field $a_{\mu}(x)$, the lattice field $\tilde{A}_{x, \mu}$ is in general neither traceless nor Hermitean. It is therefore more natural to introduce the field

$$
A_{x, \mu}^{L}=\frac{1}{2}\left[\tilde{A}_{x, \mu} V_{x, \mu}^{\dagger}+V_{x, \mu} \tilde{A}_{x, \mu}^{\dagger}\right]-\frac{1}{2 N_{f}} \operatorname{Tr}\left[\tilde{A}_{x, \mu} V_{x, \mu}^{\dagger}+V_{x, \mu} \tilde{A}_{x, \mu}^{\dagger}\right] \mathbb{1},
$$

which, by construction, is traceless and Hermitean and which transforms as

$$
A_{x, \mu}^{L \prime}=V_{x} A_{x, \mu}^{L} V_{x}^{\dagger}
$$

with the matrix $V_{x}$ located at the site $x$ on the left end of the link $(x, \mu)$. Similarly, we define the object

$$
A_{x, \mu}^{R}=\frac{1}{2}\left[V_{x, \mu}^{\dagger} \tilde{A}_{x, \mu}+\tilde{A}_{x, \mu}^{\dagger} V_{x, \mu}\right]-\frac{1}{2 N_{f}} \operatorname{Tr}\left[V_{x, \mu}^{\dagger} \tilde{A}_{x, \mu}+\tilde{A}_{x, \mu}^{\dagger} V_{x, \mu}\right] \mathbb{1},
$$

which transforms as

$$
A_{x, \mu}^{R}{ }^{\prime}=V_{x+\hat{\mu}} A_{x, \mu}^{R} V_{x+\hat{\mu}}^{\dagger},
$$

with the matrix $V_{x+\hat{\mu}}$ located at the site $x+\hat{\mu}$ on the right end of the link $(x, \mu)$. It should be noted that $A_{x, \mu}^{R}$ and $A_{x, \mu}^{L}$ are not independent but are related by parallel transport, i.e.

$$
A_{x, \mu}^{R}=V_{x, \mu}^{\dagger} A_{x, \mu}^{L} V_{x, \mu}
$$




\subsection{Constituent Quarks on the Lattice}

Using the lattice construction of a nonlinearly realized chiral symmetry presented in the previous subsection, it is now straightforward to put, for example, Georgi and Manohar's chiral quark model on the lattice. The resulting fermion action takes the form

$$
\begin{aligned}
S_{F}[\bar{\Psi}, \Psi, V, A, U] & =a^{4} \sum_{x} M \bar{\Psi}_{x} \Psi_{x}+a^{4} \sum_{x, \mu} \frac{1}{2 a}\left(\bar{\Psi}_{x} \gamma_{\mu} V_{x, \mu} U_{x, \mu} \Psi_{x+\hat{\mu}}-\bar{\Psi}_{x+\hat{\mu}} \gamma_{\mu} V_{x, \mu}^{\dagger} U_{x, \mu}^{\dagger} \Psi_{x}\right) \\
& +a^{4} \sum_{x, \mu} \frac{i g_{A}}{2 a}\left(\bar{\Psi}_{x} \gamma_{\mu} \gamma_{5} A_{x, \mu}^{L} \Psi_{x}+\bar{\Psi}_{x+\hat{\mu}} \gamma_{\mu} \gamma_{5} A_{x, \mu}^{R} \Psi_{x+\hat{\mu}}\right) \\
& +a^{4} \sum_{x, \mu} \frac{1}{2 a}\left(2 \bar{\Psi}_{x} \Psi_{x}-\bar{\Psi}_{x} V_{x, \mu} U_{x, \mu} \Psi_{x+\hat{\mu}}-\bar{\Psi}_{x+\hat{\mu}} V_{x, \mu}^{\dagger} U_{x, \mu}^{\dagger} \Psi_{x}\right) .
\end{aligned}
$$

Here $U_{x, \mu} \in S U\left(N_{c}\right)$ denotes the standard Wilson color parallel transporters living on the lattice links. It is interesting to ask if the gluon dynamics of QCD can be modeled successfully in the chiral quark model. Since the chiral quark model does not represent a systematic low-energy expansion of QCD, one should not expect to obtain quantitative results directly relevant to QCD. However, even if only qualitative insight into the success of the nonrelativistic quark model can be gained, this would be quite interesting.

The Wilson term in the above action removes the doubler fermions. As we discussed before, in standard lattice QCD this term breaks chiral symmetry explicitly. Remarkably, when chiral symmetry is nonlinearly realized, not only the fermion mass term (proportional to $M$ ) but also the Wilson term is chirally invariant. The only source of explicit chiral symmetry breaking is the current quark mass matrix $\mathcal{M}$. It is interesting to ask how the Nielsen-Ninomiya theorem [35] has been avoided. Clearly, the action of eq.(148) is local. The corresponding Dirac operator is given by

$$
\begin{aligned}
D[V, A, U]_{x, y} & =M \delta_{x, y}+\sum_{\mu} \frac{1}{2 a}\left(\gamma_{\mu} V_{x, \mu} U_{x, \mu} \delta_{x+\hat{\mu}, y}-\gamma_{\mu} V_{x-\hat{\mu}, \mu}^{\dagger} U_{x-\hat{\mu}, \mu}^{\dagger} \delta_{x-\hat{\mu}, y}\right) \\
& +\sum_{\mu} \frac{i g_{A}}{2 a}\left(\gamma_{\mu} \gamma_{5} A_{x, \mu}^{L} \delta_{x, y}+\gamma_{\mu} \gamma_{5} A_{x-\hat{\mu}, \mu}^{R} \delta_{x, y}\right) \\
& +\sum_{\mu} \frac{1}{2 a}\left(2 \delta_{x, y}-V_{x, \mu} U_{x, \mu} \delta_{x+\hat{\mu}, y}-V_{x-\hat{\mu}, \mu}^{\dagger} U_{x-\hat{\mu}, \mu}^{\dagger} \delta_{x-\hat{\mu}, y}\right) .
\end{aligned}
$$

The Nielsen-Ninomiya theorem assumes that the Dirac operator anti-commutes with $\gamma_{5}$. This is not the case when chiral symmetry is nonlinearly realized, and hence one of the basic assumptions of the Nielsen-Ninomiya theorem is not satisfied. Interestingly, Ginsparg-Wilson fermions evade the NielsenNinomiya theorem by violating the same assumption. Of course, the nonlinear realization of chiral symmetry requires an explicit pion field which is not present in the fundamental QCD Lagrangian.

Remarkably, not only the lattice fermion action but also the lattice fermion measure is gauge invariant. At first sight this seems to be a severe problem because gauge invariance of both the fermion action and the fermion measure implies that lattice fermions with nonlinearly realized chiral symmetry do not contribute to anomalies. One might even suspect that the doubler fermions have not been properly removed and thus have canceled the anomalies of the physical fermions. Fortunately, this is not the case. Indeed fermions with a nonlinearly realized chiral symmetry do not contribute to anomalies. Instead, as mentioned in the continuum discussion, the anomalies are contained in the Wess-Zumino-Witten term [144, 145] which must be added to the lattice action explicitly. 


\section{Conclusions}

In this review we have discussed some basic issues of chiral symmetry on the lattice. The recent lattice developments have put chiral symmetry on a solid theoretical basis at a nonperturbative level. As discussed in the introduction, lattice QCD can now explain nonperturbatively why nucleons can exist naturally, i.e. without fine-tuning, far below the Planck scale, provided that space-time has additional hidden dimensions. This in turn explains why gravity is so weak, a nontrivial result one obtains from lattice QCD without doing any numerical work. Even chiral gauge theories like the standard model have now been constructed rigorously beyond perturbation theory. This is a very substantial step forward in the theoretical formulation of the basic laws of Nature. The development of Ginsparg-Wilson lattice fermions is also beginning to revolutionize practical lattice QCD simulations. In particular, if new algorithmic developments go hand in hand with the recent theoretical insights, Ginsparg-Wilson fermions may lead to substantial progress towards an accurate numerical solution of QCD. As usual, many new questions arise based on the new insights. For example, supersymmetry still waits to be put on rigorously solid grounds beyond perturbation theory. Also many practical numerical calculations with Ginsparg-Wilson fermions still need to be done. We hope that we have provided a certain basis for newcomers to enter this very active field of current research.

\section{Acknowledgements}

Over the years we have discussed chiral symmetry both in the continuum and on the lattice with many colleagues including O. Bär, T. Bhattacharya, W. Bietenholz, R. Brower, G. Colangelo, N. Christ, E. Farhi, J. Gasser, M. Göckeler, J. Goldstone, M. Golterman, P. Hasenfratz, R. Jackiw, R. Jaffe, J. Jersak, D. Kaplan, A. Kronfeld, M. Laursen, H. Leutwyler, M. Lüscher, A. Manohar, T. Mehen, J. Negele, R. Narayanan, H. Neuberger, F. Niedermayer, M. Pepe, G. Schierholz, Y. Shamir, S. Sharpe, J. Smit, R. Springer, F. Steffen, and F. Wilczek. We gratefully acknowledge the insights they generously shared with us. This work was supported in part by funds provided by the U.S. Department of Energy (D.O.E.) under cooperative research agreement DE-FG02-96ER40945 and by the Schweizerischer Nationalfond (SNF).

\section{References}

[1] F. Wilczek, arXiv:hep-ph/0201222

[2] K. G. Wilson, Phys. Rev. D 10 (1974) 2445

[3] K. G. Wilson, New Phenomena In Subnuclear Physics, ed. A. Zichichi, (Plenum Press, New York), Part A (1977) 69

[4] C. G. Callan and J. A. Harvey, Nucl. Phys. B 250 (1985) 427

[5] D. B. Kaplan, Phys. Lett. B 288 (1992) 342

[6] R. Narayanan and H. Neuberger, Phys. Lett. B 302 (1993) 62

[7] R. Narayanan and H. Neuberger, Phys. Rev. Lett. 71 (1993) 3251

[8] R. Narayanan and H. Neuberger, Nucl. Phys. B 412 (1994) 574

[9] P. Hasenfratz and F. Niedermayer, Nucl. Phys. B 414 (1994) 785 
[10] T. DeGrand, A. Hasenfratz, P. Hasenfratz, and F. Niedermayer, Nucl. Phys. B 454 (1995) 587

[11] U.-J. Wiese, Phys. Lett. B 315 (1993) 417

[12] W. Bietenholz and U.-J. Wiese, Nucl. Phys. B 464 (1996) 319

[13] W. Bietenholz, R. Brower, S. Chandrasekharan, and U.-J. Wiese, Nucl. Phys. B 495 (1997) 285

[14] P. Hasenfratz, Nucl. Phys. B 525 (1998) 401

[15] P. Hasenfratz, S. Hauswirth, K. Holland, T. Jörg, F. Niedermayer, and U. Wenger, Int. J. Mod. Phys. C12 (2001) 691

[16] P. Hasenfratz, Nucl. Phys. B (Proc. Suppl.) 63 (1998) 53

[17] P. H. Ginsparg and K. G. Wilson, Phys. Rev. D 25 (1982) 2649

[18] H. Neuberger, Phys. Lett. B 417 (1998) 141

[19] H. Neuberger, Phys. Rev. D 57 (1998) 5417

[20] H. Neuberger, Phys. Lett. B 427 (1998) 353

[21] M. Lüscher, Phys. Lett. B 428 (1998) 342

[22] M. Lüscher, Nucl. Phys. B 549 (1999) 295 ; Nucl. Phys. B 568 (2000) 162

[23] A. Hasenfratz, Nucl. Phys. B (Proc. Suppl.) 119 (2003) 131

[24] M. Lüscher, JHEP 305 (2003) 052

[25] D. B. Kaplan, arXiv:hep-lat/0309099

[26] M. Creutz, Quarks, Gluons and Lattices, Cambridge Monographs on Mathematical Physics, Cambridge University Press, (1983)

[27] I. Montvay and G. Münster, Quantum Fields on a Lattice, Cambridge Monographs On Mathematical Physics, Cambridge University Press, (1994)

[28] H. J. Rothe, Lattice Gauge Theories: An Introduction, World Scientific Lecture Notes in Physics, $59(1997)$

[29] R. Gupta, Introduction to lattice QCD, published in Les Houches, "Probing the standard model of particle interactions", Part 2 (1997) 83

[30] M. Lüscher, Advanced lattice QCD, published in Les Houches, "Probing the standard model of particle interactions", Part 2 (1997) 229

[31] M. Creutz, Rev. Mod. Phys. 73 (2001) 119

[32] H. Neuberger, Ann. Rev. Nucl.. Part. Sci. 51 (2001) 23

[33] M. Salmhofer and E. Seiler, Lett. Math. Phys. 21, 13 (1991).

[34] M. Salmhofer and E. Seiler, Commun. Math. Phys. 139, 395 (1991) [Erratum-ibid. 146, 637 (1992)]. 
[35] H. B. Nielsen and M. Ninomiya, Phys. Lett. B 105 (1981) 219 ; Nucl. Phys. B 185 (1981) 20

[36] H. R. Quinn and M. Weinstein, Phys. Rev. Lett. 57 (1986) 2617

[37] L. Susskind, Phys. Rev. D 16 (1977) 3031

[38] H. S. Sharatchandra, H. J. Thun, and P. Weisz, Nucl. Phys. B 192 (1981) 205

[39] H. Kluberg-Stern, A. Morel, O. Napoly, and B. Petersson, Nucl. Phys. B 220 (1983) 447

[40] G. W. Kilcup and S. R. Sharpe, Nucl. Phys. B 283 (1987) 493

[41] S. Elitzur, Phys. Rev. D 12 (1975) 3978

[42] P. Hasenfratz and F. Niedermayer, Nucl. Phys. B 414 (1994) 785

[43] T. DeGrand, A. Hasenfratz, P. Hasenfratz, and F. Niedermayer, Nucl. Phys. B 454 (1995) 615 ; Phys. Lett. B 365 (1996) 233

[44] P. Hasenfratz, S. Hauswirth, T. Jörg, F. Niedermayer, and K. Holland, Nucl. Phys. B 643 (2002) 280

[45] M. F. L. Golterman, K. Jansen, D. N. Petcher, and J. C. Vink, Phys. Rev. D 49 (1994) 1606

[46] M. F. L. Golterman and Y. Shamir, Phys. Rev. D 51 (1995) 3026

[47] Y. Shamir, Nucl. Phys. B 406 (1993) 90

[48] V. Furman and Y. Shamir, Nucl. Phys. B 439 (1995) 54

[49] R. Narayanan and H. Neuberger, Nucl. Phys. B 443 (1995) 305

[50] Y. Kikukawa and T. Noguchi, arXiv:hep-lat/9902022

[51] L. Giusti, Nucl. Phys. B (Proc. Suppl.) 119 (2003) 149

[52] I. Horvath, Phys. Rev. Lett. 81 (1998) 4063

[53] P. Hernandez, K. Jansen, and M. Lüscher, Nucl. Phys. B 552 (1999) 363

[54] M. Golterman and Y. Shamir, Phys. Rev. D 68 (2003) 074501

[55] K. Fujikawa, Nucl. Phys. B 589 (2000) 487

[56] K. Fujikawa and M. Ishibashi, Nucl. Phys. B 605 (2001) 365

[57] W. Bietenholz, Eur. Phys. J. C 6 (1999) 537

[58] C. Gattringer, Phys. Rev. D 63 (2001) 114501

[59] C. Gattringer, I. Hip and C. B. Lang, Nucl. Phys. B 597 (2001) 451

[60] C. W. Bernard and M. F. L. Golterman, Phys. Rev. D 46 (1992) 853

[61] C. W. Bernard and M. F. L. Golterman, Phys. Rev. D 49 (1994) 486

[62] H. Wittig, Nucl. Phys. B (Proc. Suppl.) 119 (2003) 59 
[63] W. A. Bardeen, A. Duncan, E. Eichten and H. Thacker, Phys. Rev. D 59 (1999) 014507

[64] R. Frezzotti, P. A. Grassi, S. Sint and P. Weisz [Alpha collaboration], JHEP 0108 (2001) 058

[65] K. Jansen, A. Shindler, C. Urbach and I. Wetzorke [XLF Collaboration], arXiv:hep-lat/0312013.

[66] K. Fujikawa, Phys. Rev. Lett. 42 (1979) 1195

[67] K. Fujikawa, Phys. Rev. D 21 (1980) 2848 [Erratum: Phys. Rev. D 22 (1980) 1499 ]

[68] L. H. Karsten and J. Smit, Nucl. Phys. B 144 (1978) 536

[69] P. Hasenfratz, V. Laliena, and F. Niedermayer, Phys. Lett. B 427 (1998) 125

[70] F. Niedermayer, Nucl. Phys. B (Proc. Suppl.) 73 (1999) 105

[71] T. Reisz and H. J. Rothe, Phys. Lett. B 455 (1999) 256

[72] Y. Kikukawa and A. Yamada, Phys. Lett. B 448 (1999) 265

[73] K. Fujikawa, Nucl. Phys. B 546 (1999) 480

[74] H. Suzuki, Prog. Theor. Phys. 102 (1999) 141

[75] D. H. Adams, Ann. Phys. (N.Y.) 296 (2002) 131

[76] D. H. Adams and W. Bietenholz, arXiv:hep-lat/0307022

[77] M. Lüscher, Commun. Math. Phys. 85 (1982) 39

[78] E. Witten, Nucl. Phys. B 156 (1979) 269

[79] G. Veneziano, Nucl. Phys. B 159 (1979) 213

[80] B. Alles, M. D’Elia, and A. Di Giacomo, Nucl. Phys. B 494 (1997) 281

[81] L. Giusti, G. C. Rossi, M. Testa, and G. Veneziano, Nucl. Phys. B 628 (2002) 234

[82] E. Seiler and I.O. Stamatescu, "Some remarks on the Witten-Veneziano formula for the eta' mass", preprint MPI-PAE/PTh 10/87

[83] E. Seiler, Phys. Lett. B 525 (2002) 355

[84] S. Sint, Nucl. Phys. B (Proc. Suppl.) 94 (01) 79

[85] P. Hasenfratz, Nucl. Phys. B 525 (1998) 401

[86] T. Reisz and H. J. Rothe, Nucl. Phys. B 575 (2000) 255

[87] S. Capitani, Phys. Rep. 382 (2003) 113

[88] C. Alexandrou, E. Follana, H. Panagopoulos, and E. Vicari, Nucl. Phys. B 580 (2000) 394

[89] S. Chandrasekharan, Phys. Rev. D 60 (1999) 074503

[90] Y. Kikukawa and A. Yamada, Nucl. Phys. B 547 (1999) 413

[91] P. Hernandez, K. Jansen, L. Lellouch, and H. Wittig, JHEP 0107 (2001) 018 
[92] T. Blum et al., Phys. Rev. D 66 (2002) 014504

[93] T. Blum et al., arXiv:hep-lat/0007038.

[94] A. Ali Khan et al. [CP-PACS Collaboration], Phys. Rev. D 63 (2001) 114504

[95] T. W. Chiu and T. H. Hsieh, Nucl. Phys. B 673 (2003) 217

[96] T. DeGrand and U. M. Heller [MILC collaboration], Phys. Rev. D 65 (2002) 114501

[97] S. J. Dong, T. Draper, I. Horvath, F. X. Lee, K. F. Liu, and J. B. Zhang, Phys. Rev. D 65 (2002) 054507

[98] C. Gattringer et al. [BGR Collaboration], arXiv:hep-lat/0307013

[99] P. Hasenfratz, S. Hauswirth, K. Holland, T. Jorg and F. Niedermayer, Nucl. Phys. B (Proc. Suppl.) 106 (2002) 751 arXiv:hep-lat/0109007.

[100] C. Gattringer, R. Hoffmann and S. Schaefer, Phys. Lett. B 535 (2002) 358

[101] P. Hernandez, K. Jansen and L. Lellouch, Phys. Lett. B 469 (1999) 198

[102] P. Hernandez, K. Jansen, L. Lellouch and H. Wittig, Nucl. Phys. B (Proc. Suppl.) 106 (2002) 766

[103] P. Hasenfratz, S. Hauswirth, T. Jorg, F. Niedermayer and K. Holland, Nucl. Phys. B 643 (2002) 280

[104] S. Aoki et al. [CP-PACS Collaboration], Phys. Rev. Lett. 84 (2000) 238

[105] A. Ali Khan et al. [CP-PACS Collaboration], Phys. Rev. Lett. 85 (2000) 4674 ; [Erratum Phys. Rev. Lett. 90 (2003) 029902 ]

[106] Y. Aoki et al., arXiv:hep-lat/0211023.

[107] T. DeGrand [MILC Collaboration], arXiv:hep-lat/0309026

[108] N. Garron, L. Giusti, C. Hölbling, L. Lellouch, and C. Rebbi, arXiv:hep-ph/0306295

[109] A. Ali Khan et al. [CP-PACS Collaboration], Phys. Rev. D 64 (2001) 114506

[110] T. Blum et al. [RBC Collaboration], arXiv:hep-lat/0110075

[111] L. Giusti, C. Hölbling, and C. Rebbi, Phys. Rev. D 64 (2001) 114508 [Erratum: Phys. Rev. D 65 (2002) 079903 ]

[112] T. Blum, A. Soni, and M. Wingate, Phys. Rev. D 60 (1999) 114507

[113] S. Sasaki, K. Orginos, S. Ohta, and T. Blum [RBC-KEK Collaboration], Phys. Rev. D 68 (2003) 054509

[114] S. Sasaki, T. Blum, and S. Ohta, Phys. Rev. D 65 (2002) 074503

[115] D. Brommel, P. Crompton, C. Gattringer, L. Y. Glozman, C. B. Lang, S. Schaefer, and A. Schäfer [BGR Collaboration], arXiv:hep-ph/0307073

[116] S. J. Dong, T. Draper, I. Horvath, F. X. Lee, K. F. Liu, N. Mathur, and J. B. Zhang, arXiv:hep-ph/0306199 
[117] J. W. Negele, Nucl. Phys. A 711 (2002) 281

[118] P. Hagler, J. Negele, D. B. Renner, W. Schroers, T. Lippert, and K. Schilling [LHPC collaboration], Phys. Rev. D 68 (2003) 034505

[119] W. Schroers et al. [LHPC collaboration], arXiv:hep-lat/0309065

[120] M. Göckeler et al., Phys. Rev. D53 (1996) 2317

[121] M. Göckeler et al., Phys. Rev. D63 (2001) 074506.

[122] M. Göckeler et al., Phys. Rev. Lett. 92 (2004) 042002.

[123] J. Goldstone, Nuovo Cimento A 19 (1961) 154

[124] S. Coleman, J. Wess, and B. Zumino, Phys. Rev. 177 (1969) 2239

[125] C. G. Callan, S. Coleman, J. Wess, and B. Zumino, Phys. Rev. 177 (1969) 2247

[126] S. Weinberg, Physica 96A (1979) 327

[127] J. Gasser and H. Leutwyler, Ann. Phys. (N.Y.) 158 (1984) 142 ; Nucl. Phys. B 250 (1985) 465

[128] H. Georgi, Weak Interactions and Modern Particle Theory, Benjamin-Cummings Publishing Company, (1984)

[129] J. Gasser, M. E. Sainio, and A. Svarc, Nucl. Phys. B 307 (1988) 779

[130] E. Jenkins and A. Manohar, Phys. Lett. B 255 (1991) 558

[131] V. Bernard, N. Kaiser, J. Kambor, and U.-G. Meissner, Nucl. Phys. B 388 (1992) 315

[132] T. Becher and H. Leutwyler, Eur. Phys. J. C 9 (1999) 643

[133] D. B. Kaplan, M. J. Savage, and M. B. Wise, Phys. Lett. B 424 (1998) 390 ; Nucl. Phys. B 534 (1998) 329

[134] P. F. Bedaque, H.-W. Hammer, and U. van Kolck, Phys. Rev. C 58 (1998) 641 ; Nucl. Phys. A 676 (2000) 357

[135] P. F. Bedaque and U. van Kolck, Ann. Rev. Nucl. Part. Sci. 52 (2002) 339

[136] S. Myint and C. Rebbi, Nucl. Phys. B 421 (1994) 241

[137] I. A. Shushpanov and A. V. Smilga, Phys. Rev. D 59 (1999) 054013

[138] R. Lewis and P.-P. A. Ouimet, Phys. Rev. D 64 (2001) 034005

[139] H. M. Müller, S. E. Koonin, R. Seki, and U. van Kolck, Phys. Rev. C 61 (2000) 044320

[140] J.-W. Chen and D. B. Kaplan, arXiv:hep-lat/0308016

[141] D. J. Lee and I. C. F. Ipsen, arXiv:nucl-th/0308052

[142] S. Chandrasekharan, M. Pepe, F. D. Steffen, and U.-J. Wiese, arXiv:hep-lat/0306020

[143] A. Manohar and G. W. Moore, Nucl. Phys. B 243 (1984) 55 
[144] J. Wess and B. Zumino, Phys. Lett. B 37 (1971) 95

[145] E. Witten, Nucl. Phys. B 223 (1983) 422 ; Nucl. Phys. B 223 (1983) 433

[146] H. Georgi and A. Manohar, Nucl. Phys. B 234 (1984) 189 